\section{TRES NUEVAS DEFIXIONES ROMANAS EN SU CONTEXTO ARQUEOLÓGICO. LA NECRÓPOLIS DE LLANOS DEL PRETORIO (CÓRDOBA) ${ }^{1}$}

\section{THREE NEW ROMAN DEFIXIONES IN THEIR \\ ARCHAEOLOGICAL CONTEXT. THE NECROPOLIS OF LLANOS DEL PRETORIO (CÓRDOBA)}

\author{
SERGIO GARCÍA-DILS DE LA VEGA \\ UNIVERSIDAD DE SEVILLA \\ 凶: sergio.garcia.dils@icloud.com \\ MANUEL RUBIO VALVERDE \\ UNIVERSIDAD DE GRANADA \\ 凶: manuelrubiovalverde@gmail.com
}

\section{RESUMEN}

Se presentan tres nuevas defixiones romanas halladas, en su contexto arqueológico, en la necrópolis de Llanos del Pretorio, un nuevo sector de la Necrópolis Septentrional de colonia Patricia Corduba excavado a finales del año 2016. En este ámbito, con una cronología de uso centrada en época julio-claudia, han sidos documentados, total o parcialmente, hasta quince recintos funerarios. En las líneas que siguen se estudiarán dos de ellos, en cuyo interior se localizaron las tres tabellae.

Palabras clave: epigrafía romana, defixio, necrópolis, Hispania Baetica.

\section{ABSTRACT}

In this paper are presented three new Roman defixiones, found in their archaeological context inside the necropolis of Llanos del Pretorio, a new sector of the Northern Necropolis of colonia Patricia Corduba excavated at the end of the year 2016. In this area, with a chronology of use centered in the Julio-Claudian period, have been documented, totally or partially, up to fifteen funerary enclosures. In the following lines, will be studied two of them, within which the three tabellae were located.

Keywords: Roman epigraphy, defixio, necropolis, Hispania Baetica.

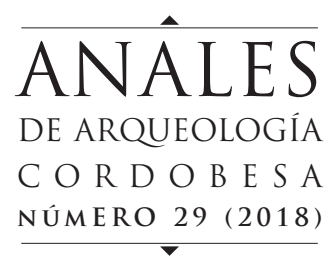

C O R D O B E S A

\section{ANALES} (n)

(1) 


\section{CONTEXTO ARQUEOLÓGICO DEL HALLAZGO}

El descubrimiento de este nuevo sector de necrópolis, en el que se hallaron tres defixiones, se produjo durante un Seguimiento Arqueológico dirigido por uno de nosotros (M.R.V.) en los números 1 y 3 de la Avenida Llanos del Pretorio de la ciudad de Córdoba, parcela situada a unos $400 \mathrm{~m}$ al norte del perímetro amurallado de época romana (Fig. 1). El solar en cuestión, de unos 820 $\mathrm{m}^{2}$, ya fue objeto de una Actividad Arqueológica Preventiva entre septiembre de 2006 y febrero de 2007 (Penco, 2007), y de un primer Seguimiento Arqueológico entre agosto y diciembre de 2008 (Penco, 2008). Ambas intervenciones pusieron de manifiesto una secuencia cronológica que arrancaba en época romana altoimperial, con posteriores ocupaciones medievales, modernas y contemporáneas. Con respecto a la fase altoimperial, la que más interés tiene para este trabajo, se documentaron algunas estructuras a las que no fue posible asignar una funcionalidad precisa. Durante ese primer Seguimiento Arqueológico se llevó a cabo la

\footnotetext{
2 Restos pertenecientes a la Necrópolis Septentrional se han documentado en multitud de intervenciones arqueológicas durante estos últimos años. Se pueden destacar, entre otras muchas, las desarrolladas en la antigua fábrica de la Constancia (Vaquerizo et alii, 2005), en Santa Rosa (Moreno, 2007), en la calle Abderramán I (Salinas, 2015), en los Jardines de la Agricultura (Clapés et alii, 2015), o en varios solares de la Avenida de las Ollerías (Baena, 1989; Penco et alii, 1993; López, 2006).

3 El paralelo más cercano lo encontramos en la vía funeraria localizada por el arqueólogo Agustín López en la Avenida de las Ollerías (López, 2006), igualmente de finalidad rigurosamente funeraria, y a la que también abrían fachada, aunque solamente por su margen meridional, varios recintos funerarios señalados mediante cipos de calcarenita (Vaquerizo y Sánchez, 2008: 123).
}

construcción de los muros pantalla, pero no se llegó a completar la excavación de todo el solar, pues antes de finalizar el vaciado completo del mismo, la construcción se paralizó, quedando el solar abandonado hasta que en 2016 una nueva promotora decidió retomar las construcciones en el mismo. Desde la Gerencia Municipal de Urbanismo de Córdoba, tras la solicitud de la promotora, se emitió una nueva información urbanística de carácter arqueológico, en la que se le requirió un nuevo Seguimiento Arqueológico. Fue durante este segundo Seguimiento Arqueológico cuando fue documentado un amplio sector de necrópolis perteneciente a la Necrópolis Septentrional de colonia Patricia.

Este nuevo sector, de unos $270 \mathrm{~m}^{2}$, viene a sumarse a otros sectores de esta misma necrópolis exhumados durante los últimos años en diferentes intervenciones arqueológicas ${ }^{2}$, que han aportado una cronología de uso para esta Necrópolis Septentrional desde el siglo I d.C. hasta el siglo VI d.C. (Salinas, 2015: 270), uso que evidentemente no es sincrónico en todas sus zonas, siendo una buena prueba de ello este nuevo sector, con una cronología centrada, a priori, en época julio-claudia.

Esta zona de necrópolis, en la que se han documentado, total o parcialmente, hasta 15 recintos funerarios, se articula a partir de una vía secundaria, con una finalidad estrictamente funeraria -via sepulcralis-, que da acceso a los diferentes recintos, que se localizan, en batería, a ambos lados de la misma ${ }^{3}$. Se ha documentado un tramo de $28 \mathrm{~m}$ de esta vía funeraria, con una anchura de unos 2,40 m -aproximadamente unos 8 p.r.-, y que presenta un firme de picadura de calcarenita. Según la orientación que sigue, 


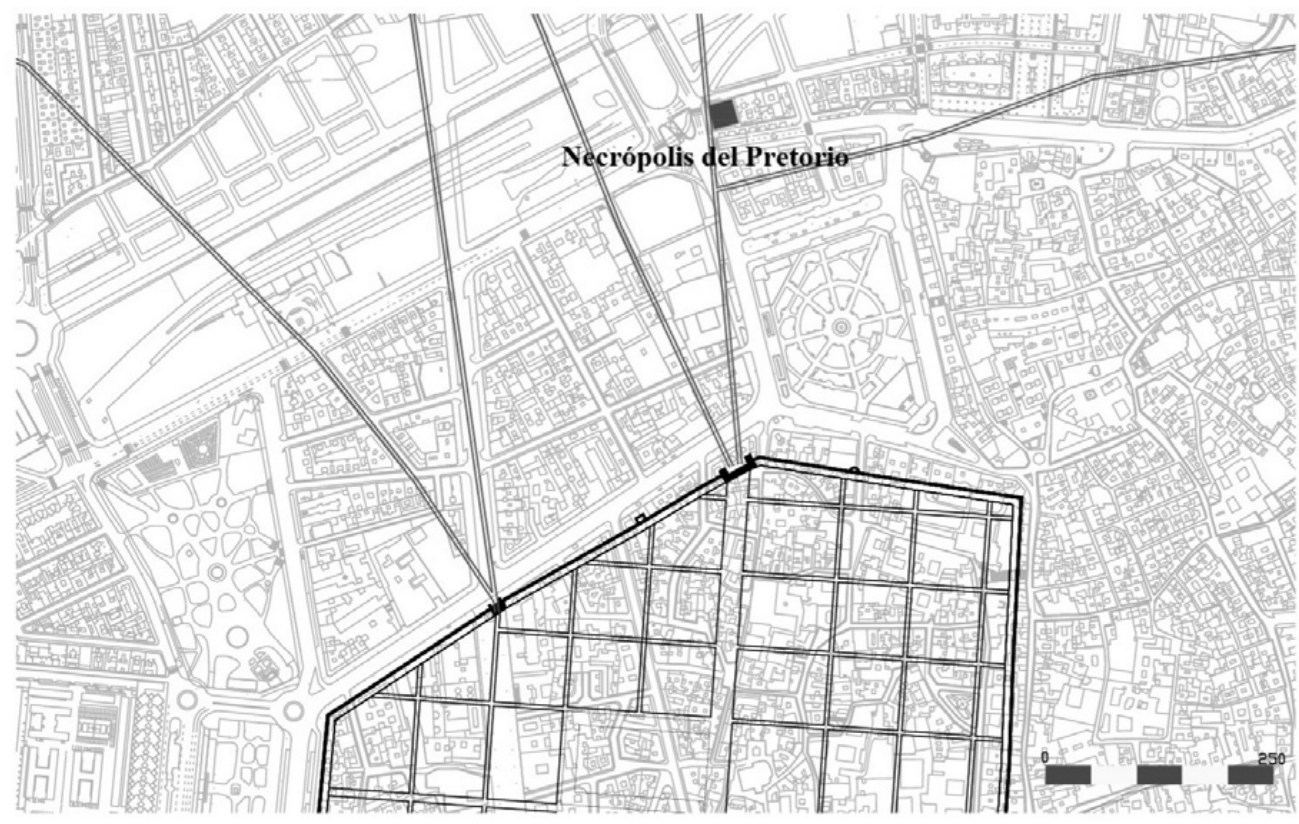

Fig. 1: Situación de la necrópolis con respecto al recinto amurallado de época romana (M. Rubio).

en sentido este-oeste, muy probablemente se trate de una vía perpendicular al conocido como Camino del Pretorio, vía que partía de la Porta Praetoria, situada en la muralla norte de la ciudad, de la que posiblemente tomó el nombre que se ha conservado hasta nuestros días (Melchor, 1995: 162 ss.), y fosilizada en parte de las actuales avenida Llanos del Pretorio y avenida del Brillante 4 .

Este sector funerario queda cerrado, al sur, por un gran muro, de $70 \mathrm{~cm}$ de ancho, que alterna la mampostería y la sillería. Inmediatamente al norte de éste se sitúa una primera batería de recintos, en la que se han documentado, total o parcialmente, un total de 9 acotados funerarios -Recintos $A, B, C$, $D, E, F, G, H$ e I-, todos, creemos, con un tamaño estándar de $12 \times 12$ p.r., 3,55 × 3,55 $\mathrm{m}$. En este sentido, destaca el hallazgo, in situ, en dos de estos recintos, de sendos ci- pos de calcarenita con la inscripción $L \cdot P$ - XII -I(ocus) p(edum) XII-, indicatio pedaturae que alude precisamente al tamaño de los mismos, 12 × 12 p.r. ${ }^{5}$. Inmediatamente al norte de esta primera batería de recintos

4 En concreto, se trata de la vía denominada Servitus via susum ad Montes Societatis Sisaponensis, que se adentraba hacia la Sierra, Ilegando a cruzar los ríos Guadiato y Guadamuño antes de la confluencia de éstos, y cuyo principal objetivo fue facilitar la entrada de cobre y plomo argentífero procedentes de un importante complejo minero en uso durante los siglos I y II d.C., de ahí, que durante el siglo III esta vía se mantuviera con una finalidad exclusivamente funeraria (Vaquerizo, 2001: 135; Melchor, 1995: 162 ss.; Moreno, 2007: 41-42).

5 Estas dos indicationes pedaturae vienen a unirse a la decena que, hasta este momento, se conocían en la ciudad. En este sentido, destaca también la intervención de Agustín López en Ollerías, en la que se documentaron hasta tres indicationes pedaturae, también in situ, que siguen la misma fórmula que las documentadas en esta intervención (Ruiz Osuna, 2007: 31, Tabla 1; Vaquerizo y Sánchez, 2008: 123). 
se sitúa la vía funeraria a la que hacíamos mención más arriba (vid. supra), y al norte de la misma, una segunda batería de recintos, en la que se han documentado un total de cinco recintos funerarios - Recintos $\mathrm{J}, \mathrm{K}$, $\mathrm{L}, \mathrm{M}$ y $\mathrm{N}-$, de los que tres de ellos siguen la misma modulación de los anteriores, $12 \mathrm{x}$ 12 p.r., mientras que dos de ellos -Recintos $\mathrm{L}$ y $\mathrm{M}$ - ocupan una zona que en un primer momento no fue concebida como espacio para enterramientos, pues presenta el mismo firme que la vía funeraria. Sin embargo, ese gran espacio, de unos $15 \mathrm{~m}$ de largo -poco más de 50 p.r.-, se dividió en dos partes iguales, y acabó siendo usado como espacio funerario, quizá como consecuencia de la demanda de más espacio para enterramientos. Al norte de esta segunda batería de enterramientos se localizaría una tercera, de la que en la intervención se documentó parte de otros dos posibles recintos -Recintos 0 y $\mathrm{P}$-, manteniendo el segundo de ellos la modulación de 12 x 12 p.r. Aunque es escasa la superficie documentada del Recinto $P$, es suficiente para plantear la existencia de otra vía funeraria, paralela a la documentada en la intervención, que se situaría al norte de este recinto, pues presenta muros de mampostería que cierran sus laterales sur, este y oeste, por lo que el acceso al mismo debía situarse al norte. Por lo tanto, las dos primeras baterías de recintos darían fachada a la vía documentada durante nuestra intervención, mientras que los recintos de la tercera batería de recintos darían fachada a esta vía que creemos se situaría más al norte. Además, hemos de destacar el hallazgo, en la mayor parte de los recintos, de un ustrinum en el que se llevarían a cabo las cremaciones de los individuos posteriormente enterrados en los mismos. Estos ustrina, situados normal- mente en la zona central del recinto, se caracterizan por tratarse de una simple fosa en el terreno que presenta sus bordes rubefactados, y que en algunos casos presentaban muros de mampostería que cerraban alguno de sus laterales. Seguramente de carácter familiar, en varios de ellos se han hallado los restos de varias cremaciones superpuestas, separadas entre sí por capas de arcilla o cal.

Se ha documentado un total de 67 enterramientos, que se reparten de la siguiente forma: 50 enterramientos de cremación -dos de ellos dobles-, 11 inhumaciones perinatales, 2 inhumaciones de sendos perros, 2 posibles inhumaciones perinatales -se documentaron los contenedores funerarios, dos ánforas cortadas longitudinalmente, pero no restos humanos- y 2 posibles cremaciones -de una de ellas solamente se documentó el ajuar que la acompañaba, y de la otra el contenedor funerario, una urna cerámica, pero no restos humanos-. Más de la mitad de estos enterramientos iban acompañados de su correspondiente ajuar, destacando la presencia mayoritaria de ungüentarios vítreos, aunque la variedad de ajuares es extensa bullae, alguna moneda, colgantes, etc.-.

Atendiendo al contexto concreto de las defixiones, hemos de señalar que aparecieron en dos recintos funerarios diferentes (Fig. 2), por lo que pasamos a comentar en profundidad cada uno de ellos.

La defixio A apareció en el estrato de colmatación del Recinto Funerario $\mathrm{H}$, situado en la batería de recintos sur. Este estrato, denominado U.E. 34, está conformado por arciIla y abundantes fragmentos cerámicos, un fragmento de hueso trabajado, algunos restos metálicos -concretamente un clavo de hierro y un fragmento indeterminado de bronce-, y 


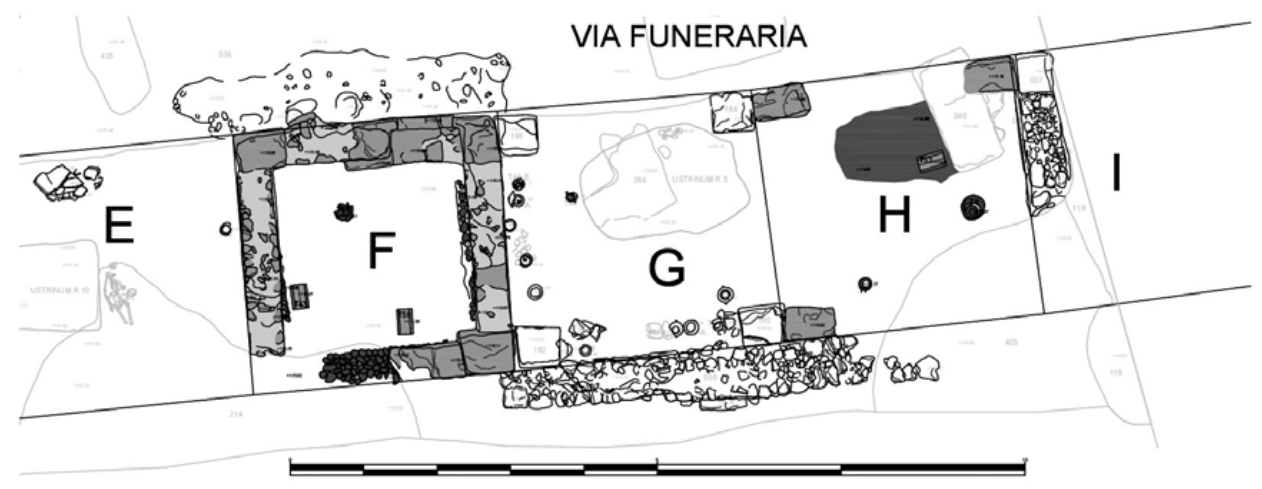

Fig. 2: Detalle de la planta de los Recintos Funerarios F y H (Dibujo: J. M. Tamajón).
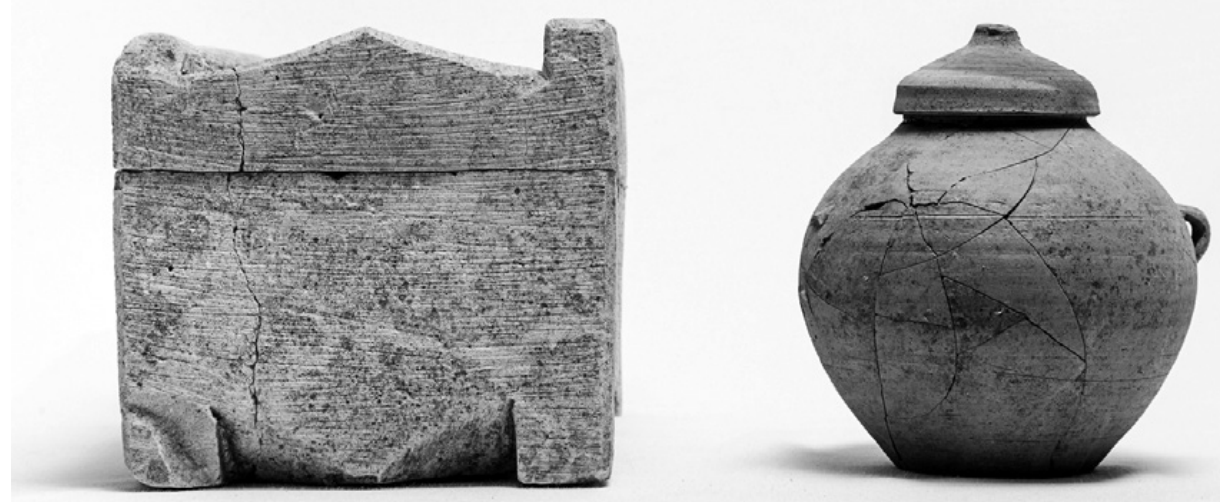

Fig. 3: Urnas cinerarias completas documentadas en el interior del Recinto Funerario H (Fotografía: J.

Rojas).

restos óseos animales -se han documentado restos óseos de perro y, sobre todo, burro y caballo- ${ }^{6}$. En relación a la cerámica recuperada en este estrato, destaca la aparición de un solo fragmento de Terra Sigillata Itálica y de Terra Sigillata Gálica, varios fragmentos de cerámica tipo Peñaflor, un fragmento de la forma Mayet XXXVII de paredes finas, varios fragmentos de cerámica vidriada romana, concretamente de un skyphos de la forma 2 de López Mullor, un fragmento de barniz rojo pompeyano, una lucerna derivada de Dressel 3 , y varios fragmentos de cerámica de tradición ibérica, conjunto que arroja una cro-

6 Agradecemos al Dr. Rafael M. Martínez Sánchez, responsable del estudio arqueofaunístico dentro del proyecto "Sepulcretum Llanos del Pretorio", su deferencia al compartir con nosotros los primeros resultados de su estudio, aún en proceso y por lo tanto absolutamente provisionales. 
nología provisional de Tiberio-Claudio7. Con respecto al recinto funerario, cuenta con un tamaño de $12 \times 12$ pies, y estaba delimitado, a priori, por cuatro cipos de calcarenita, de los que solamente se han conservado tres, pues la zona donde se situaría el cuarto de ellos, la esquina suroriental del recinto, fue afectada por una fosa de cronología contemporánea. En su interior se localizó un ustrinum, que presentaba los restos de una única cremación, y un total de tres enterramientos -Enterramientos 6, 7 y 52- (Fig. 3), aunque la defixio no apareció en relación directa con ninguno de ellos. Se trata de tres cremaciones secundarias que fueron depositadas en dos urnas cerámicas y en una urna de piedra.

Las defixiones $\mathrm{B}$ y $\mathrm{C}$ aparecieron en la colmatación del Recinto Funerario F, situado también en la batería de recintos sur. Este estrato, denominado U.E. 144, está confor-

\begin{tabular}{|c|c|c|c|c|c|c|c|c|c|c|c|c|c|}
\hline \multicolumn{14}{|c|}{ MATERIALES RECUPERADOS EN LA UE. 38 (TIBERIO-CLAUDIO) } \\
\hline & $\begin{array}{l}B^{8} 5 \\
\text { GEN. }\end{array}$ & \begin{tabular}{|c|} 
B \\
27 \\
GEN.
\end{tabular} & $\begin{array}{c}\text { B } \\
72 \\
\text { GEN. }\end{array}$ & $\begin{array}{l}\text { B } 79 \\
\text { GEN. }\end{array}$ & $\begin{array}{l}\text { B } 90 \\
\text { GEN. }\end{array}$ & $\begin{array}{l}\text { B } 5 \\
\text { SEL. }\end{array}$ & $\begin{array}{c}\text { B } \\
19 \\
\text { SEL. }\end{array}$ & \begin{tabular}{|l|} 
B 33 \\
SEL.
\end{tabular} & $\begin{array}{l}\text { B } 35 \\
\text { SEL. }\end{array}$ & $\begin{array}{l}\text { B 55 } \\
\text { SEL. }\end{array}$ & $\begin{array}{c}\text { B } \\
177 \\
\text { GEN. }\end{array}$ & $\begin{array}{c}\text { B } \\
181 \\
\text { GEN. }\end{array}$ & TOTAL \\
\hline Común diag. & 18 & 5 & 3 & 6 & & & & & & & & & 32 \\
\hline Común no diag. & 30 & 18 & 25 & 28 & & & & & & & & & 101 \\
\hline T. S. I. & & 1 & & & & & & & & & & & 1 \\
\hline T. S. G. & & 1 & & & & & & & & & & & 1 \\
\hline Tipo Peñaflor & 9 & & 2 & & & & & & & & & & 11 \\
\hline Paredes finas & 26 & & 4 & & 1 & & & & & 1 & & & 32 \\
\hline B. R. Pompeyano & 1 & & & & & & & & & & & & 1 \\
\hline $\begin{array}{l}\text { Cerámica de Trad. } \\
\text { Ibérica }\end{array}$ & & & 6 & 2 & 4 & & & & & & & & 12 \\
\hline Vidriada romana & & & & & & & & & 10 & & & & 10 \\
\hline Lucerna & & 1 & 1 & 1 & & & 1 & & & & & & 4 \\
\hline $\begin{array}{l}\text { Ungüentario } \\
\text { cerámico }\end{array}$ & 1 & 2 & 1 & & & 2 & & & & & & & 6 \\
\hline Ánfora & 1 & 1 & & 3 & & & & & & & & & 5 \\
\hline Botella espatulada & 1 & & & & & & & & & & & & 1 \\
\hline Otros & & & & & & & & $1^{9}$ & & & $2^{10}$ & $1^{11}$ & 4 \\
\hline
\end{tabular}

Tabla resumen de los elementos documentados en la U.E. 38 -no se incluyen los restos óseos animales-.

7 En este caso, también agradecemos la atención de Liliana Hernández Lozano y Sonia Vargas Cantos, ceramólogas del proyecto "Sepulcretum Llanos del Pretorio", al compartir los resultados preliminares de su trabajo, aún en desarrollo.

8 B: Bolsa; GEN: Inventario General; SEL: Inventario Material Selecto.

9 Un fragmento de hueso trabajado.

10 Un fragmento informe de bronce y un fragmento de escoria de metal.

11 Un clavo de hierro. mado por arcilla y abundantes fragmentos cerámicos, cinco clavos de hierro, una moneda y un ungüentario de vidrio muy fragmentado. En relación a la cerámica recuperada en este estrato, destaca la aparición de las formas Drag. 15/17 y Drag. 29 de Terra Sigillata Gálica, varios fragmentos de Marmorata, dos bordes de cerámica de tradición ibérica, o un borde la forma Martinez I en cerámica tipo 

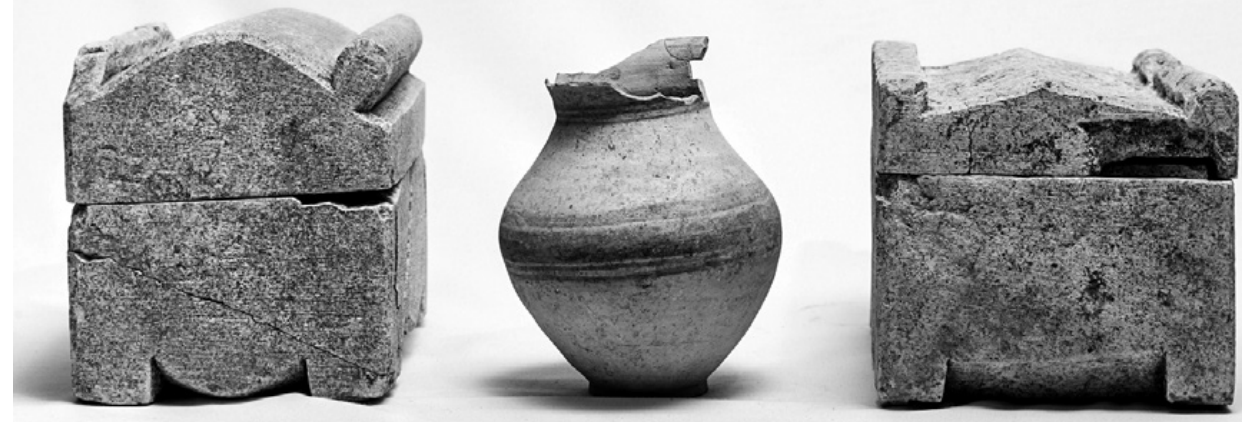

Fig. 4: Urnas cinerarias documentadas en el interior del Recinto Funerario F (Fotografía: J. Rojas).

Peñaflor, que aportan una cronología provisional centrada en época de Claudio, que podría extenderse hasta época de Nerón por la presencia de la Marmorata ${ }^{12}$. Por su parte, el ungüentario de vidrio pertenece al tipo Isings 6 o De Tomasso 7, fechado desde época de Augusto hasta finales del siglo I d.C. (Isings, 1957: 22). Los rasgos arcaicos del mismo, con una división bastante marcada entre el cuello y el depósito, aquilatan su cronología a la primera mitad del siglo I d.C. (Sánchez de Prado, 2018: 217) ${ }^{13}$. El recinto, de un tamaño de 12 x 12 p.r., estaba delimitado, a priori, por cuatro cipos de calcarenita, de los que solamente se han conservado tres, pues la zona donde se situaría el cuarto de ellos, el de la esquina suroccidental, fue afectada por una fosa para desechos de época califal, en la que destaca el hallazgo de un individuo en decúbito prono. Uno de los cipos que lo delimitan, el de la esquina sur, presenta la ins- cripción $L \cdot P \cdot X I I-I$ (ocus) p(edum) XII-, que alude, como señalábamos más arriba (vid. supra), al tamaño del recinto, en el que la realidad se corresponde a la perfección con lo recogido en la inscripción. En este caso, los laterales del recinto fueron cerrados con muros de mampostería sobre cimentaciones de cantos rodados, muros que presentan un cuidado remate interior, con un revestimiento de mortero de cal que presenta incisiones que pretenden imitar un despiece de sillería. En el lateral norte se localiza la entrada al recinto, modestamente "monumentalizada" a partir de la colocación de un quinto cipo central que la flanquea junto con el de la esqui-

12 Vid. supra n. 7.

13 Las Dras. Almudena Velo-Gala y Chloe Duckworth, y David Govantes, han sido los responsables del estudio del material vítreo dentro del proyecto "Sepulcretum Llanos del Pretorio". Los autores agradecen su deferencia al compartir con nosotros los primeros resultados de su estudio. 
na nororiental, con un escalón de entrada en piedra calcarenita que presenta una quicialera, por lo que este recinto estaría cerrado con una puerta. En su interior se localizaron un total de tres enterramientos -Enterramientos 43, 48 y 59- (Fig. 4), aunque la defixio no apareció en relación directa con ninguno de ellos. Se trata de tres cremaciones secundarias que fueron depositadas en dos urnas de piedra y en una urna cerámica.

\section{LAS DEFIXIONES}

Se presentan a continuación las tres defixiones halladas en la intervención arqueológica, que se suman a la reducida nómina de esta tipología epigráfica que han sido localizadas en Baetica ${ }^{17}$. Al innegable interés que presenta su contenido, se suma la valiosa información que aporta el estudio de su contexto arqueológico, que viene a enriquecer nuestro conocimiento sobre el fenómeno de las tablillas de maldición en entornos necropolitanos ${ }^{18}$.

\section{LA DEFIXIO A}

Se trata de una lámina de plomo de forma irregular, recortada prácticamente a $90^{\circ}$ en el ángulo superior derecho, de 9,26 cm de ancho y 5,14 cm de alto, con $3 \mathrm{~mm}$ de grosor máximo (Fig. 5). Contiene el siguiente texto:

\begin{tabular}{|c|c|c|c|c|c|c|c|}
\hline \multicolumn{7}{|c|}{ MATERIALES RECUPERADOS EN LA UE. 144 (CLAUDIO-NERÓN) } \\
\hline & $\begin{array}{c}\text { B 89 } \\
\text { GEN. }\end{array}$ & $\begin{array}{c}\text { B 123 } \\
\text { GEN. }\end{array}$ & $\begin{array}{l}\text { B 153 } \\
\text { GEN. }\end{array}$ & $\begin{array}{c}\text { B 26 } \\
\text { SEL. }\end{array}$ & $\begin{array}{c}\text { B 46 } \\
\text { SEL. }\end{array}$ & $\begin{array}{c}\text { B 72 } \\
\text { SEL. }\end{array}$ & TOTAL \\
\hline Común diagnosticable & 3 & & & & & & 3 \\
\hline Común no diagnosticable & 15 & 13 & & & & & 28 \\
\hline T. S. G. & 4 & & & & 1 & & 5 \\
\hline T. S. G. Marmorata & & & & & 7 & & 7 \\
\hline Tipo Peñaflor & 1 & 2 & & & & & 3 \\
\hline Cerámica de Trad. Ibérica & & 4 & & & & & 4 \\
\hline Otros & & & 514 & $11^{15}$ & & 116 & 7 \\
\hline
\end{tabular}

Tabla resumen de los elementos documentados en la U.E. 144.

14 Cinco clavos de hierro.

15 Una moneda.

16 Un ungüentario de vidrio.

17 Antes de la presente publicación, se habían localizado y editado en Baetica dieciséis textos de estas características, exhumados en once localizaciones distintas, de un total de veintiocho en el conjunto de Hispania. Para una visión general sobre la cuestión en la provincia, vid. ORDÓÑEZ y GARCÍA-DILS, e.p. 2018.

18 Además de las obras clásicas de A. Audollent (1904) y K. Preisendanz (1972), vid. un estado de la cuestión reciente y autorizado sobre este fenómeno en Alfayé, 2009 y Sánchez Natalías, 2012.
AVGII - GLVCIIRAII SABBINIS FAVSTI

FORTVNATAII NICOCLIS PRIMAII

NICIIROTIS MACIIDONIS INGIINVI

GALLAII PHILARGVRI DIONVSI

CHITIIRIDIS NICONIS AVGIINIS CHRIISTI

MARITVMI BASILI MAGIIIAII

Auge, Glucerae, Sabbinis, Fausti, / Fortunatae, Nicoclis, Primae, / Nicerotis, Macedonis, Ingenui, / Gallae, Philarguri, Dionusi, I Chiteridis, Niconis, Augenis, Chresti, / Maritumi, Basili, Mageiae. 

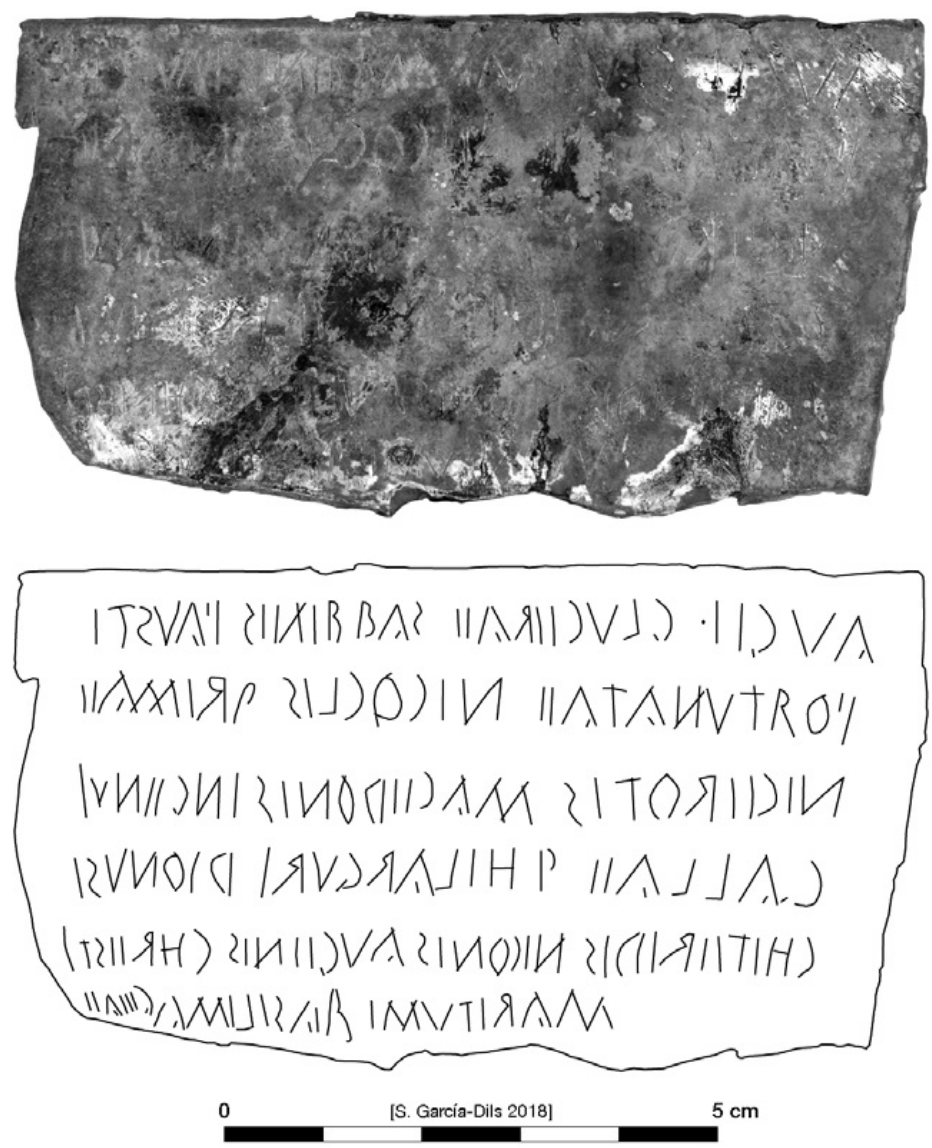

Fig. 5: Defixio A (S. García-Dils).

El texto está escrito de derecha a izquierda, confirmando la superposición de los trazos correlativos de los diferentes caracteres que fueron escritos también en ese orden. Prácticamente todas las letras presentan esta orientación, salvo en el caso de los caracteres con lóbulo, como las $B$ de Sabbinis y BasiIi, la $R$ de Fortunatae y las $P$ de Primae y Philarguri. Las $A$ alternan el trazo interior de derecha a izquierda y a la inversa. I longa al final de I. 3 y 5. Los caracteres, en las II. 1-4, tienen una altura uniforme de entre 4,0 y $4,5 \mathrm{~mm}$, que desciende a entre 3,0 y
3,5 $\mathrm{mm}$ en II. 5-6, reduciéndose hasta los $2,0 \mathrm{~mm}$ al final de I. 6 . En cuanto al interlineado, es de $3,5 \mathrm{~mm}$ entre las $\| .1$ y 2 , de $6,0 \mathrm{~mm}$ entre las II. 2 y 3 , de $3,0 \mathrm{~mm}$ entre las II. 3 y 4 , de $4,0 \mathrm{~mm}$ entre las II. 4 y 5 , oscilando entre 2,0 y $3,5 \mathrm{~mm}$ entre las II. 5 y 6 . Solamente hay una interpunción en el texto, de forma circular, marcada con rotundidad siguiendo al primer nombre, Auge. Los antropónimos están bien ordenados y separados, no cortándose ninguno a final de línea. La escritura, como suele ser habitual en las defixiones, empieza ordenada, y se va desor- 
denando a medida que avanza el texto y se acaba el espacio libre en el soporte, lo que se hace especialmente evidente en los dos últimos nombres, Basili y Mageiae. También se adapta a grietas e irregularidades, especialmente en la I. 6, donde el texto presenta un amplio margen a la derecha, para salvar una protuberancia de la lámina.

Tras la mención de un personaje femenino, Auge, en nominativo, encabezando la defixio, le sigue una sucesión de diecinueve antropónimos, todos en caso genitivo, distribuidos regularmente en seis líneas. Son los siguientes:

Auge (I. 1). Antropónimo femenino de origen griego (Aüץn - Fraser y Matthews, 19872008: s.v.; Abascal, 1994: 292; Lörincz y Redö, 1994: 225; Solin, 1996: 349-350; Lozano, 1998: 43) e inspiración mitológica $^{19}$. En Roma, más de la mitad de las portadoras del cognomen Auge son esclavas o libertas (Solin, 2003: 577-579).

19 Se entienda bien como una de las ${ }^{\top} \Omega$ pa (Hyg. Fab. 183.5.2), o bien como la hija de Aleo, rey de Tegea (Arcadia), madre del héroe Télefo tras unirse a Heracles (Ov. H. 9.49; Hyg. Fab. 101.1.1; Hyg. Fab. 252.1.1).

20 "Teidiae Auges" (CIL VI $9319=$ CIL VI 33803); "Fla(via)e Auges" (CIL VI 18295); "Auges alumna[e ---]" (CIL VI 29546); "Lebisiniae Auges” (EE VIII, 1899, n²5).

21 Por ejemplo, y sin ánimo de exhaustividad, "Corneliae M(arci) f(iliae) Auge" (CIL VI 16359); "Iuliae Auge" (CIL XIV 1180); "Iuniae Auge" (CIL VI 35629); "Galgestiae Auge" (CIL V 163); "Aemiliae Auge" (CIL VI 11125); "Magniae T(iti) f(iliae) Auge" (CIL VI 14450); "Cocceiae Auge" (CIL VI 15893); "Cordiae P(ubli) I(ibertae) Auge" (CIL VI 16089).

22 Siguiendo la lectura original de Rodrigo Caro, Clodia Lucera, A. Canto considera que se debe desechar el posible paralelo registrado en un sarcófago de Medina Sidonia, que CIL II 1320 corregía por Clodia [G]lucera (HEp 2005, 116).

23 Sobre los nombres personales de origen semita en la Hispania romana, vid. López Castro y Belmonte, 2012.
Se trata de un nombre muy común, que cuenta con sendos paralelos en inscripciones funerarias documentadas en Baetica, uno de ellos en la misma Corduba ( $\mathrm{ClL}$ $\left.I^{2} / 7,418\right)$ y el segundo en Gades (IRPCádiz 371).

En cuanto al caso en el que aparece el antropónimo, se trata claramente del nominativo. Cabría argumentar al respecto que, si bien la forma canónica del nombre en genitivo sería Auges, solamente disponemos de un puñado de paralelos epigráficos para este uso ${ }^{20}$, mientras que, en cambio, nos ha llegado un buen número de ejemplos de la misma forma Auge empleada tanto en dativo como en genitivo ${ }^{21}$. Sin embargo, teniendo en cuenta que en el mismo texto, en I. 5, aparece la flexión en -enis de este nombre en genitivo, Augenis, se puede afirmar que aquí estamos ante un nominativo.

Glucera (I. 1) (= Glycera). De nuevo, se trata de un cognomen de mujer de proceden-

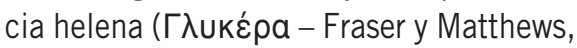
1987-2008: s.v.; Abascal, 1994: 381; Solin, 1996: 454; Lozano, 1998: 99; Lörincz, 1999: 168). La mitad de los casos registrados en Roma corresponden a esclavas o libertas (Solin, 2003: 943944). Se trata del primer ejemplo con esta grafía localizado en el conjunto de Hispania ${ }^{22}$. En su forma canónica, Glycera, se puede citar un único caso hispano, la liberta Paccia Glycera, de Emerita Augusta (CIL II 5275).

Sabbis (I. 1). Nombre femenino de origen semítico (Lörincz, 2002: 39; Solin, 2014a: 34; Solin, 2014b: 245), que se documenta por primera vez en Hispania23. En general, poco frecuente, con contados 
paralelos centrados sobre todo en Roma, como Aninia L. I. Sabbis (CIL VI 11665), Tarquitia Sabbis (CIL VI 27114), Vibia Sabbis (CIL VI 28886), Fabia Sabbis (CIL VI 28109) y Antonia M. I. Sabbis (CIL VI 26153). También en algunos puntos de Italia, como Servilia P. I. Sabbis, de Formia (AE 1995, 273); Salvidena C. I. Sabbis, de Amiternum ( $A E$ 1992, 473); la liberta Octavia Sabbis, de Verona (CIL V 3690); Varia L. I. Sabbis, de Capua (CIL X 4391).

Faustus (I. 1). Antropónimo masculino (Kajanto, 1965: 29-30, 41, 72-73, 134, 272; Duthoy, 1989; Abascal, 1994: 359; Lörincz, 1999: 136), con numerosos testimonios en Baetica. En la misma Corduba, contamos con la estela funeraria de un gladiador myrmillo contrarete (CIL II/7, $361=A E$ 1962, 48); P. Argentarius D. I. Faustus (CIL II2/7, $415=$ HEp 2008, 56); un niño de siete meses de ese nombre (C/L II2/7, 547); también aparece en una defixio fechada en la segunda mitad del siglo I a.C. $\left(C / L I^{2} / 7,252=A E 1934\right.$, 24).

Fortunata (I. 2). Cognomen de mujer, de los más extendidos en Hispania y, en general, en el conjunto del Imperio (Kajanto, 1965: 13-14, 18, 29-30, 72, 93, 273; Duthoy, 1989; Abascal, 1994: 31, 371; Lörincz, 1999: 150-151). En la ciudad solamente se ha documentado una Fortunata ( CIL II2/7, 463), si bien contamos con una veintena de ejemplos conocidos en el conjunto de Baetica. Por ejemplo, Messia Fortunata, de Naeva (CIL II 1079 = CILA II.1, $275=$ AE 2012, $737=$ HEp 2012, 427); una serva de Osset (CIL II $1257=$ CILA II.2, 588); Cornelia Fortu- nata, de Gades (CIL II 1797); la liberta Mummia Fortunata, de Ostippo (C/L II/2/5, $967=$ CIL II $1449=$ CILA II.4, 1145).

Nicocles (I. 2). No contamos con paralelos epigráficos para este cognomen de varón de procedencia helena, pese a estar muy extendido en su lengua original (Nıкок入nิৎ - Fraser y Matthews, 1987-2008: s.v.; Abascal, 1994; Solin, 1996; Lozano, 1998; Lörincz, 2000; no registrado en Solin, 2003) ${ }^{24}$. Consta un ejemplo de su forma femenina Nicoclea (Nıкок $\lambda \dot{\varepsilon}$ - Solin, 2003: 122), en Roma (CIL VI 26578).

Prima (I. 2). Nombre femenino ampliamente documentado en el conjunto del mundo romano (Kajanto, 1965: 29-30, 73-78, 134, 291; Abascal, 1994: 465; Lörincz, 2000: 161-162). Sólo en Corduba, contamos con tres libertas con este cognomen, Annaea L. I. Prima (ClL II2/7, 406), Cor-

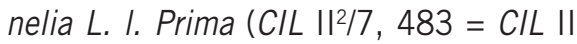
2286) y Albucia M. I. Prima (CIL $\|^{2} / 7$, 555).

Niceros (I. 3). Nombre masculino, de pro-

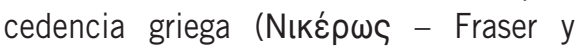
Matthews, 1987-2008: S.v.; Abascal, 1994: 438; Solin, 1996: 218; Lozano, 1998: 142; Lörincz, 2000: 100). Más de la mitad de los conocidos en Roma son esclavos o libertos (Solin, 2003: 120 121). Nos ha llegado evidencia epigráfica de sendos paralelos en Baetica, localizados en Magacela $\left(C / L ~ I^{2} / 7,967=H E p\right.$ 1994, $155=$ AE 1997, 792) y Hornachos ( $E E \mathrm{IX}, 181$ ) respectivamente.

24 Sí disponemos, en cambio, de testimonios en las fuentes literarias latinas. Por citar un par de ejemplos, "tyrannum Nicoclem" (Cic. Off. 2.81.4); "Timarchus Nicoclis filius" (Plin. HN 11.167.4). 
Macedo (I. 3). Cognomen heleno de varón

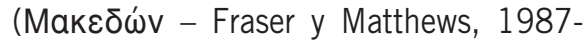
2008: s.v.; Abascal, 1994: 407; Solin, 1996: 367-368; Lozano, 1998: 128; Lörincz, 2000: 42; Solin, 2003: 639641). Tenemos conocimiento de un par de paralelos hispanos, ambos localizados en Tarraco, el liberto Aurelius Macedo (CIL II $4182=$ = CIL II2/14, 1105) y VIpius Maced(o), que dedica una lápida funeraria a su contubernalis, veterano de la Legio VII Gemina Felix (CIL II²/14, $1082=A E$ 1966, 191).

Ingenuus (I. 3). Antropónimo masculino muy extendido en el conjunto del Imperio (Kajanto, 1965: 314-315; Abascal, 1994: 390; Lörincz, 2000: 194), que cuenta con un testimonio en la propia Corduba, el essedarius Ingenuus (CIL II/7, $362=$ $A E$ 1952, 126 = AE 1962, 49). También se ha registrado en la ciudad en su forma femenina, Ingenua (CIL II2/7, $472=\mathrm{Cl}$ II 2283).

Galla (I. 4). Cognomen femenino (Kajanto, 1965: 12, 45, 51, 195; Abascal, 1994: 377; Lörincz, 1999: 160), con un ejemplo en Corduba, Quintia P. f. Galla (CIL II/7, $271=$ CIL II 5522).

Philargurus (I. 4) (= Philargyrus). Nombre

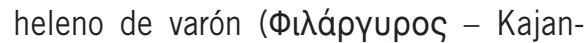
to, 1965: 13; Fraser y Matthews, 19872008: s.v.; Abascal, 1994: 455-456; Solin, 1996: 420-421; Lozano, 1998: 158; Lörincz, 2000: 137). Dos terceras partes de los conocidos en Roma son esclavos 0 libertos (SOLIN, 2003: 815-818). Cuenta con numerosos paralelos en el conjunto del Imperio, aunque no así en Baetica, con un único ejemplo, L. Valerius Philargurus, de Gades (CIL II 1905 = IRPCádiz
301). Sí consta en Corduba la variante femenina, la liberta Philargyris (CIL II2/7, 419 = CIL II 2259). Otra variante en Lusitania, M. Villius M. I. Philargurius, de Aldea del Obispo (HEp 2012, $165=A E$ $1983,498)$. Un par de paralelos más en Hispania Citerior, L. Minucius Philargurus, de Tarraco (CIL II2/14, $1624=$ CIL II 4391) y un Philargurus en una defixio de Emporiae (HEp 1994, 447 = AE 2004, 834).

Dionusius (I. 4) (= Dionysius). Antropónimo

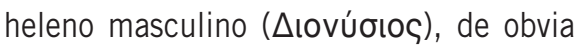
conexión mitológica, que aparece por primera vez documentado en esta forma en Hispania (Fraser y Matthews, 1987-2008: S.v.; Abascal, 1994: 344; Solin, 1996: 276-278; Lozano, 1998: 74-75; Lörincz, 1999: 101-102; Solin, 2003: 323-329). En su forma canónica, se trata de uno de los nombres griegos más populares en el mundo romano (Solin, 2009: 82), uno de ellos en Baetica, T. Ann(ius) Dionysius, de Aratispi $\left(C / L ~ I^{2} / 5,733=\right.$ CIL II 2056). También se han registrado sendas variantes femeninas en Baetica, la esclava Dionisia, en una defixio de Corduba (CIL $I^{2} / 7,250=$ HEp 2000, $163=$ AE 1934, 23 = AE 2014, 648; Sánchez Natalías, 2014) y Iulia Dionissia en Tucci (CIL II/2/5, $107=$ CIL II $5473=$ CILA III.2, $436=$ HEp 1995, 474 = AE 1965, 81). En el resto del Imperio sí se documentan algunos ejemplos más de la forma Dionusius, como Dionusius Pulli Q. s., de Minturnae (CIL | 2682), o L. Mundicius L. I. Dionusius, de Ortygia (CIL III $7223=$ CIL III $7230=C / L \mid 2251)$.

Chiteris (I. 5) (= Cytheris). Cognomen femenino de procedencia griega (KuOnpís - Fra- 
ser y Matthews, 1987-2008: S.v.; Solin, 1996: 263; Lörincz, 1999: 90; no registrado en Abascal, 1994; Lozano, 1998), que suele interpretarse en clave mitológi$\mathrm{ca}^{25}$. Dos terceras partes de las conocidas en Roma son esclavas o libertas (Solin, 2003: 634-635). La forma registrada en la defixio presenta varias irregularidades. En primer lugar, i por $y$; además, se produce una transferencia de la $h$ de Cytheris a ch. Para esta grafía, disponemos de cuatro paralelos conocidos, Macriana つ. I. Chiteris, de Roma (CIL VI 33602); Chiteris, de Verona (CIL V 3497); Peticia P. I. Chiteris, de Villa San Sebastiano (CIL IX $3824=$ CIL | 1771 = EE VIII.1, 166); la liberta Plania Chiteris, de Narbo (CIL XII 5058). Sí contamos con un testimonio hispano para la forma canónica, Cornelia A. I. Cytheris, de Saguntum (CIL II2/14, 437a = HEp 2009, $448=$ AE 2009, 658).

Nico o Nicon (I. 5). Nombre masculino de origen heleno (Nıкú o Níkwv - Fraser y Matthews, 1987-2008: S.v.; Abascal, 1994: 438; Solin, 1996: 443; Lozano, 1998: 143; Lörincz, 2000: 100-101; SOLIN, 2003: 906-907). En Baetica, un L. C(ornelius?) Nicon, de Burguillos del Cerro (CIL II $5357=$ EE IX 146). En Lusitania, conocemos a [Phaedi]mus Daphnus Nico (CIL II 512) y Licinius Nicon (HEp 1999, 91), ambos de Emerita. En la Hispania Citerior, un sello sobre sigiIlata de Nico, de Tortosa ( $A E$ 1995, 522); Pomp(eius, onio) Nico, de Caesaraugusta (HEp 1990, 738); Nico, de Novallas (HEp 2010, 422).

Auge (I. 5). De nuevo, el cognomen femenino Auge, con una flexión del genitivo en -enis ${ }^{26}$.
Chrestus (I. 5). Nombre masculino de origen heleno (Xрn̂бтос - Fraser y Matthews, 1987-2008: s.v.; Abascal, 1994: 326; Solin, 1996: 470; Lozano, 1998: 58; Lörincz, 1999: 54). La mitad de los casos registrados en Roma corresponden a esclavos o libertos (Solin, 2003: 10041006). Cognomen bien documentado en el conjunto del Imperio, con ligeras variaciones. Con esta misma grafía, en Baetica conocemos a un Chrestus (HEP 1997, 29) y a Titin(ius) Chrestus (HEp 1994, $189=$ AE 1991, $1013=$ = AE 1992, 914), ambos de Ugultunia; otro Chrestus, de Malaca ( $A E$ 1981, 509). También $M$. V() C(h)restus, de Lebrija (EE IX 212a = CILA II.3, 1007). En la misma Corduba, se ha registrado el posible paralelo de $N$. Abull[ius] Chr\{i\}est[us] (CIL II/7, $396=$ CIL II 2254), aunque también podría reconstruirse con opciones menos frecuentes, como Chr\{i\}est[os] o Chr\{i\}est[inus].

Maritumus (l. 6). Cognomen masculino para el que disponemos de sendos ejemplos en Hispania Citerior (Kajanto, 1965: 308; Abascal, 1994: 415; Lörincz, 2000: 58).

25 Efectivamente, en general se relaciona el nombre Cytheris con uno de los epítetos de Afrodita, Cytherea

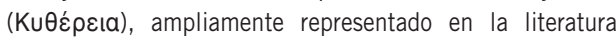
latina -por ejemplo, Verg. Aen. 1.257, 1.657, 4.128, $5.800,8.523,8.615-$. No se debe descartar tampoco un carácter estrictamente geográfico, en referencia a la isla de Citera (KúӨnpa), por lo demás uno de los lugares asociados al nacimiento de la diosa (Hes. Theog. 176 ss.).

26 Disponemos de varios paralelos epigráficos de la flexión del genitivo de Auge en -enis: "Muttienae Q(uinti) I(ibertae) Augeni[s]", de Teanum Apulum (AE 1976, 154); "Folliae Augenis", de Aquinum (AE 1978, 110); "Augenis", de Osijek (CIL III 3289); "[An]niae L(uci) I(ibertae) Augenis" (CIL VI 5369), "Eergenniaes(!) C(ai) I(ibertae) Augenis" (CIL VI 23916) y "Prociliae Augenis", las tres de Roma (CIL VI 25070); "Vareniae Augenis", de Nemausus (CIL XII 4004); Augenis, de Puteoli (AE 2007, 408). 
En primer lugar, en el listado de nombres de Grandas de Salime, interpretado como censo, donde aparece dos veces el nombre Maritumus (HEp 2005, 21 = HEp 2009, $21)^{27}$; también en una inscripción de Valentia, donde se alude a un Maritumus y su hija, [M]arituma (CIL II2/14, $72=\mathrm{ClL}$ II $6005=$ HEp 1996, $979=A E 1995$, 968). A estos testimonios, cabría añadir también una Marituma en Castulo (CIL II 3311 = CILA III.1, 152).

Basilius (I. 6). Nombre de varón de origen

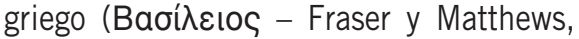
1987-2008: s.v.; Abascal, 1994: 299; Lörincz y Redö, 1994: 273; Solin, 1996: 493; no registrado en Lozano, 1998; Solin, 2003: 1087-1088), que aparece por primera vez en Baetica. Nos ha llegado un paralelo lusitano, Saturius Basilius, del territorium de Caurium (CIL II 797). En Hispania Citerior, en este caso como gentilicio, M. Basilius, de Saguntum (CIL $\|^{2} / 14,514=H E p 1990,715=H E p$ $1993,390=A E 1986,455)$.

Mageia (I. 6). Nombre femenino de origen celta (Giorcelli, 2007; no registrado en Kajanto, 1965; Abascal, 1994; Lörincz, 2000) que se documenta por primera vez en Hispania. Solamente contamos con dos paralelos de este cognomen, localizados ambos en la Transpadana, en unas inscripciones localizadas en Novaria ( $A E$ $2010,603)$ y Vercellae (AE 2007, 649), respectivamente. Una posibilidad alternativa, aunque poco probable, sería interpretar aquí un cognomen más habitual, Ma-

27 Por sus características formales, dejando aparte que la inscripción se realizó sobre una losa de pizarra, quizá podría plantearse la caracterización de este peculiar texto como una defixio. gia (Kajanto, 1965; Abascal, 1994: 409; Lörincz, 2000: 46), en el que se habría usado el diptongo ei en lugar de $\bar{i}$, un arcaísmo lingüístico extendido en la epigrafía republicana hispana (Díaz, 2008: 24).

En el texto no se aprecia ningún patrón en particular, sucediéndose indistintamente nombres masculinos (11) y femeninos (9), de origen heleno (12), latino (6), celta (1) 0 semítico (1), sin agruparse ni distribuirse de ninguna forma definida que nos ilustre sobre el carácter de la inscripción. En este sentido, ante la ausencia de verbos o signa magica, su interpretación como defixio se desprende de su contexto -funerario-, el soporte empleado -una lámina de plomo- (Sánchez Natalías, 2011), y la dirección de la escritura -de derecha a izquierda-. Los nombres están correctamente escritos, sin omitir caracteres, y con su flexión en genitivo realizada de forma canónica. Solamente cabe señalar como peculiaridades la sustitución de y por $u$, así como la transferencia de la $h$ al escribirse Cytheris como Chiteris.

Todos los antropónimos están bien documentados, portados en general por individuos de extracción servil (Kajanto, 1965; Lozano, 1998; Solin, 1996; 2003; 2014b). Se registran tanto cognomina de carácter mitológico -Auge, Dionusius y Chiteris-, como derivados de adjetivos, en general con un deseo positivo o relacionados con el carácter o cualidades -reales o deseadas- del esclavo -Glucera, Faustus, Fortunata, Philargurus, Chrestus, Basilius- (Kajanto, 1965: 71-73). También los hay relativos al orden de nacimiento -Prima- (Kajanto, 1965: 73-78), a la condición social -Ingenuus- (Kajanto, 1965: 313315) e, incluso, de tipo geográfico -Macedo, Galla, Maritumus-, sin que ello implique 
necesariamente que se recoja el origen real del personaje (Kajanto, 1965: 195, 308). Por fin, algunos pueden corresponder tanto a personajes históricos o literarios, como estar relacionados con la propia etimología del antropónimo -Nicocles, Niceros, Nico-.

En el caso del nombre con el que finaliza el texto, Mageia, dado el carácter de este documento epigráfico, resultaría atractivo bus-

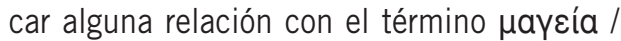
mageia. Sin embargo, desechamos esta op- ción considerando que se encuentra, como el resto, en caso genitivo, Mageiae, y sobre todo dado que no se trata de un término registrado en defixiones.

Aunque pudiera resultar sugerente, también descartamos aquí interpretar el comienzo de la inscripción, auge, como imperativo de augeo, opción que permitiría establecer un sentido positivo para la tabella de "aumenta -la riqueza, la felicidad, etc.- de Glucera, etc.".
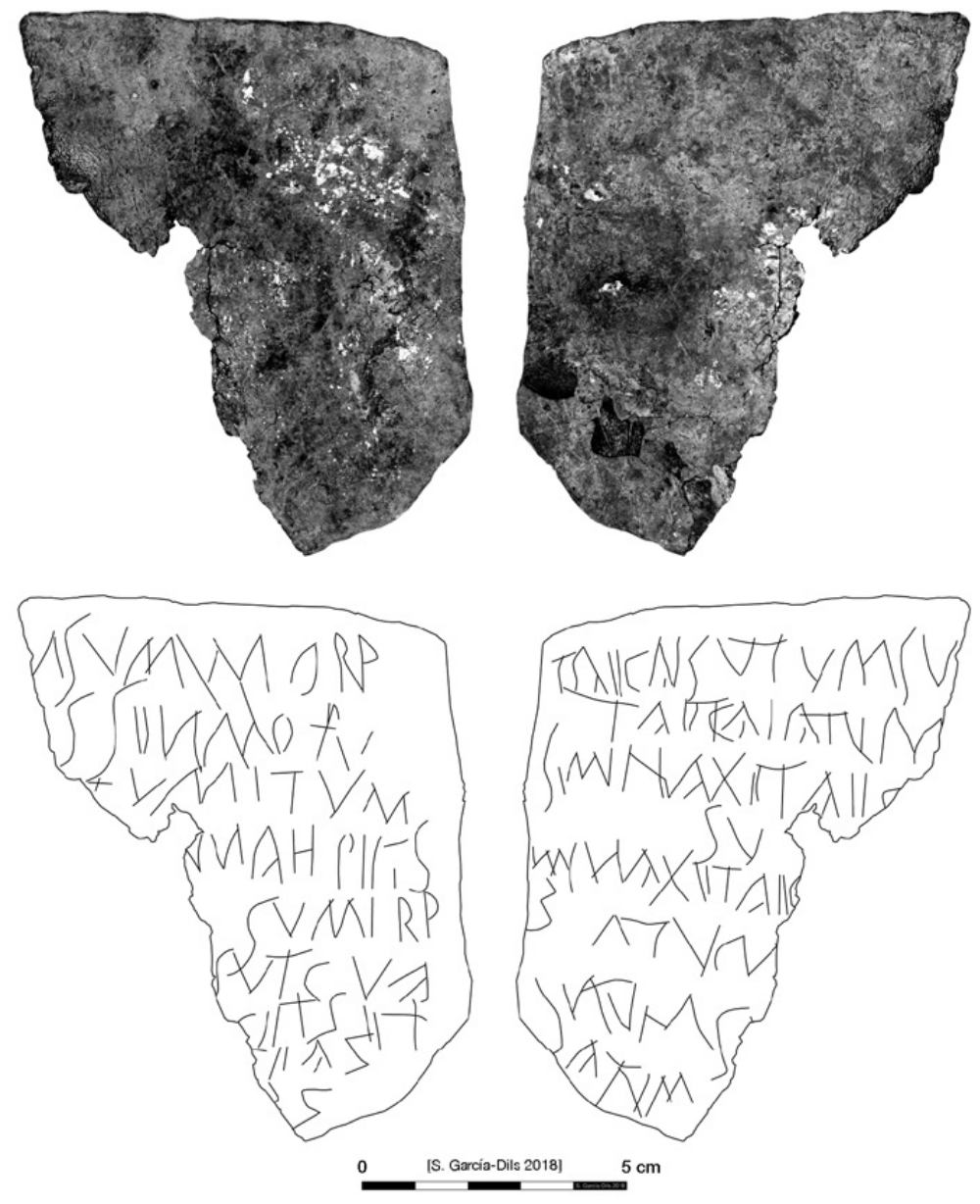

Fig. 6: Defixio B (S. García-Dils). 


\section{LA DEFIXIO B}

La pieza consiste también en una lámina plúmbea de morfología irregular, en este caso opistógrafa, con el ángulo superior derecho más definido, recortado prácticamente a $90^{\circ}$. Tiene unas dimensiones de $(8,56) \mathrm{cm}$ de ancho y $10,28 \mathrm{~cm}$ de alto, con $2 \mathrm{~mm}$ de grosor máximo, faltando aproximadamente un tercio de la tabella (Fig. 6). Se observa claramente el orificio dejado por un clavo. El texto inscrito es el que sigue:

\section{Cara A}

PROMIMVS M[VTVS SIT]
VT OMNIIS S[INT MVTI]
MVTI MVT[I ---]
STIIPHANV[S ---]
PRIMVS [---]
AVCTVS [---]
TIISTII+[---]
SAIIT[---]
S+[---]
Cara B
[---]VS MVTVS IACIIAT
[---] MVTA IA\{T\}CIIAT
[--- IA]CIIAT IIXANIMIS
[---]VS
[--- IA]CIIAT IIXANIMI
[---] MVTA 'S'
[---]+S MVTVS
[---] MVTA

$P<h>r o^{\prime} n^{7}$ imus m[utus sit] / ut omnes s[int muti] / muti mut[i ---] / Stephanu[s ---] / Primus [---] / Auctus [---] / testes [---] / SAET[---] / S[---] // [---]us mutus iaceat / [---] muta ia\{t\}ceat / [--- ia]ceat exanimis / [---] us / [---ia]ceat exanimis / [---] muta / [---]us mutus / [---] muta.

De nuevo, el texto está inscrito de derecha izquierda, estando los caracteres tam- bién escritos en ese orden, tal como muestra la superposición del ductus de los trazos correlativos. En este caso, el inscriptor no intentó hacer el esfuerzo de orientar las letras hacia la izquierda, salvo en tres casos la $N$, tal como puede observarse en las II. 2, 4 y 14 , así como en la $N$ transformada en $M$ en I. 1. La irregularidad de la superficie del soporte, así como la evidente falta de pericia del defigens, hace que la caligrafía sea deplorable.

En la I. 1 de la cara A, se advierte claramente que el trazo que convertía en una $M$ una $N$ trazada al revés está grabado después, con un ancho mayor, ejerciendo más presión y un recorrido totalmente recto, de manera que inicialmente se habría escrito PRONIMVS, corrigiendo después -erróneamente- a PROMIMVS. El resto de las $M$ del texto presentan un trazo izquierdo de recorrido curvo. Las irregularidades que presenta la lámina en I. 2 hacen que el grabado de los caracteres sea más impreciso, prolongándose algunos trazos hasta la I. 1 al haberse ejercido más presión, como en la $M$ y la primera S. En I. 3, antes de la $T$ hay un par de trazos que da la sensación de que han sido tachados con el mismo punzón que se utilizó para grabar la inscripción. En I. 7, + es un trazo curvo, que sube en diagonal de izquierda a derecha, compatible con una $S$. En las II. 7, 8 y 9 , debido a la falta de espacio, se graban $S$ más esquemáticas, que en I. 8 , dada la angulosidad del carácter, acaso pudiera tratarse de una $Z$.

En cuanto a la cara B, en la I. 11 el defigens escribió inicialmente IATEAT, superponiendo después una $C$ a la $T$ para corregir por IACEAT. En I. 15, se ha trazado la $S$ final de la I. 14, por falta de espacio. En I. 16, cabe 
interpretar + como $V$, correspondiente a un nombre masculino en nominativo.

Los caracteres, en las II. 1-4, tienen una altura de entre 9,0 y $11,0 \mathrm{~mm}$, que se reduce a entre 7,0 y $8,5 \mathrm{~mm}$ en las II. 5-9 en la cara $A$, prolongándose las $s$ hasta los 19,5 $\mathrm{mm}$, con un interlineado en general reducido de entre 2,5 y 4,0 mm en las II. 1-6, superponiéndose prácticamente las II. 6-9. La cara B presenta unos caracteres más uniformes, de entre 7,5 y 9,5 mm en todas las líneas, con $s$ de hasta $16,5 \mathrm{~mm}$, mientras que el interlineado varía entre la superposición y los $3,5 \mathrm{~mm}$.

Desafortunadamente, como ya se ha indicado, nos falta aproximadamente un tercio de la tabella. Con toda probabilidad, seguiría el mismo tenor en ambas caras, recogiéndose una sucesión de nombres masculinos y femeninos, seguidos de la correspondiente fórmula de maldición. Los antropónimos son los siguientes:

$\boldsymbol{P}<\boldsymbol{h}>$ ronimus (I. 1). Nombre masculino de origen griego (Фрovífuos - Fraser y Matthews, 1987-2008: S.v.; Abascal, 1994: 457 y 472; Solin, 1996: 403; Lozano, 1998: 166; Lörincz, 2000: 140). La mitad de los registrados en Roma pueden caracterizarse como esclavos o libertos (Solin, 2003: 758-759). Se debe leer aquí Phronimus, cognomen para el que contamos con dos paralelos en la Hispania Citerior, Aemilius Phronimus, de Saguntum (CIL II2/14, 710 = CIL II 3985), y L. Iunius C. Prhonimu[s], de Jérica, si bien confundiendo Phr por Prh (CIL II2/14, $245=$ CIL II 3995). Para la grafía $\sin h$, contamos con sendos paralelos en Roma, el liberto C. Popilius Pronimus (CIL VI 16392) y Pronim(us) (CIL XV 5439); en
Pompeya, Pronimus (CIL IV 3092) y A. Messius Phronimus, que aparece como Pronimus en varias tablillas del archivo de Iucundus (CIL IV 3340, 28, 32, 55, etc.). En su versión femenina (Фpoví $\mu \eta)$, un paralelo bético para esta grafía, Val(eria) Pron(i)m(a), de Italica (ERItálica $163=$ CILA II.2, 24). Cabe interpretar la transcripción de Фрovíuos por Pronimus como un rasgo de arcaísmo lingüístico habitual en la epigrafía republicana hispana (Díaz, 2008: 24-25).

Stephanus (I. 4). Antropónimo de varón de procedencia helena ( $\Sigma$ tغ́фavoc - Fraser y Matthews, 1987-2008: s.v.; Abascal, 1994: 516; Solin, 1996: 548-550; Lozano, 1998: 181-182; Lörincz, 2002: 94; Solin, 2003: 1267-1272). Cuenta con un paralelo cordubense, el sacerdote Aurelius Stephanus (CIL II2/7, 234 = CIL II 5521 $=A E$ 1962, 267). Además, también en Baetica, conocemos otros paralelos, aunque presenten un estado fragmentario, $A$. Plot[ius] Stepha[nus], de Gades (CIL II 1865 = IRPCádiz 00261); [S]tep(h)anus, de Olaurum (CIL II/2/5, $953=$ CILA II.4, 1176); M. Publicius Stepha[nus], de Ossigi $\left(C / L\left\|^{2 / 7}, 11=C / L ~\right\| 3352=C I L A\right.$ III. 1, 343).

Primus (I. 5). Nombre masculino muy extendido (Kajanto, 1965: 29-30, 73-78, 134, 291; Abascal, 1994: 467; Lörincz, 2000: 161-162). Dos paralelos en la misma Corduba, C. Octavius T. C. I. Primus (CIL $\|^{2} / 7,341=C / L$ II 2238) y L. Cornelius Primus (CIL II/7, $483=$ CIL II 2286).

Auctus (I. 6). Cognomen masculino de uso frecuente (Kajanto, 1965: 350; Duthoy, 1989; Abascal, 1994: 291; Lörincz y Red, 1994: 220). En Corduba, tenemos 
testimonios del praeceptor L. Lollidius Auctus (CIL II2/7, 340) y de P. Cornelius Philoclis I. Auctus (CIL II2/7, $384=A E$ 1978, 00404); en su versión femenina, de Luclena Aucta (C/L II2/7, $402=$ HEp 2001, 259 = HEp 2010, 123).

Se trata claramente de un ejemplar de defixio iudiciaria, según la ya clásica categoría definida por A. Audollent (1904: 471472), en la que se recurre a lo sobrenatural en busca de una ventaja judicial, maldiciendo al otro litigante, así como a sus testigos ${ }^{28}$. Nos encontramos con una tipología bien representada entre las execraciones depositadas en contextos necropolitanos, por delante de las agonísticas y eróticas (Sánchez Natalías, 2012: 123). Efectivamente, el corpus de defixiones iudiciariae engloba 67 testimonios griegos y 46 latinos y, en general, las tabellae de este tipo eran colocadas, mayoritariamente en contexto funerario -como es nuestro caso-, en el transcurso de los preparativos del juicio (Gager, 1992: 117; Marco, 2010a: 410). En estos casos, se insiste especialmente en contrarrestar la deposición de testimonio por parte de la otra parte litigante, para lo que disponemos de ejemplos muy explícitos en las defixiones documentadas. Audollent (1904) ya recogía algunos ejemplos significativos en los que se incide precisamente en la mudez de abogados defensores y testigos $-n^{0} 111=n^{0} 112=C / L$ XIII $11069=$ CIL XIII $11070=A E$ 1897, 50; $\mathrm{n}^{\circ} 134=A E$ 1901,$184 ; n^{0} 219 ; n^{0} 220 ; n^{0} 222 ; n^{0} 303-$ (GAGER, 1992: 116-150). En este sentido, resulta especialmente ilustrativa una maldición encontrada en la necrópolis romana de

28 Sobre los matices en la caracterización de estos textos y la propuesta de distinción entre defixiones iudiciariae y prayers for justice, vid. Versnel, 1991; 2010.
Praunheim/Frankfurt a. M., en la que se pide a los dioses del Inframundo que un cierto [Ma?]rius Fronto, cuando sea llamado frente al gobernador, sea incapaz de presentar testimonio contra un tal Sextus, insistiéndose en el texto repetidamente sobre esta cuestión -"Rogo manes / inferi ut [Ma?]/rius Fronto $a[d v] /$ ersarius Sext[i] / sit vanus neq/ue loqui pos/[s]it contra / [S]extum, ut / Fronto fiat / mutus qu/[um] (=cum) access/[e]rit // consular/[e]m, ut sit / mutus ne/que possit / loqui ne/que qui[c]/quam ag[e]/re, tanqu/am nullo / ab inf[e]/ris"- (AE 1978, 545 = AE 2011, 832; Marco, 2010a: 411-412; Blänsdorf y Scholz, 2011). Sobre esta práctica, cabe citar también en contextos epigráficos similares la mención de Muta Tacita, diosa infernal del silencio (Marco, 2010b), en dos defixiones judiciales halladas respectivamente en Kempten ( $A E 1958,150=A E 2010,109)$ y Sisak ( $A$ IJ $557=A E$ 1921, $95=A E$ 2008, 1080 = AE 2010, 109; Barta, 2017).

En la provincia Baetica disponemos también de notables testimonios adscribibles a esta tipología. En primer lugar, en la propia Corduba, sendas tabellae que fueron halladas juntas en el interior de una urna cineraria procedente, presuntamente, de la calle Abéjar, en el área definida como la Necrópolis Oriental de la ciudad (Ventura, 1996; CIL $\left.\|^{2} / 7,251 a\right)$. Como en el caso que nos ocupa, contiene varias menciones expresas de la privación de la palabra de los testigos, "Priamus I(ibertus) mutus sit / omnibus modis", además de "silient" (= "sileant") y "omut[e] sq[ua]nt" (= "obmutescant"). Hay que señalar, además, que las similitudes de los caracteres entre estas defixiones y la que se presenta ahora son realmente notables, tanto en su tipología como en el trazado, por lo que incluso podría proponerse que fueran obra de 


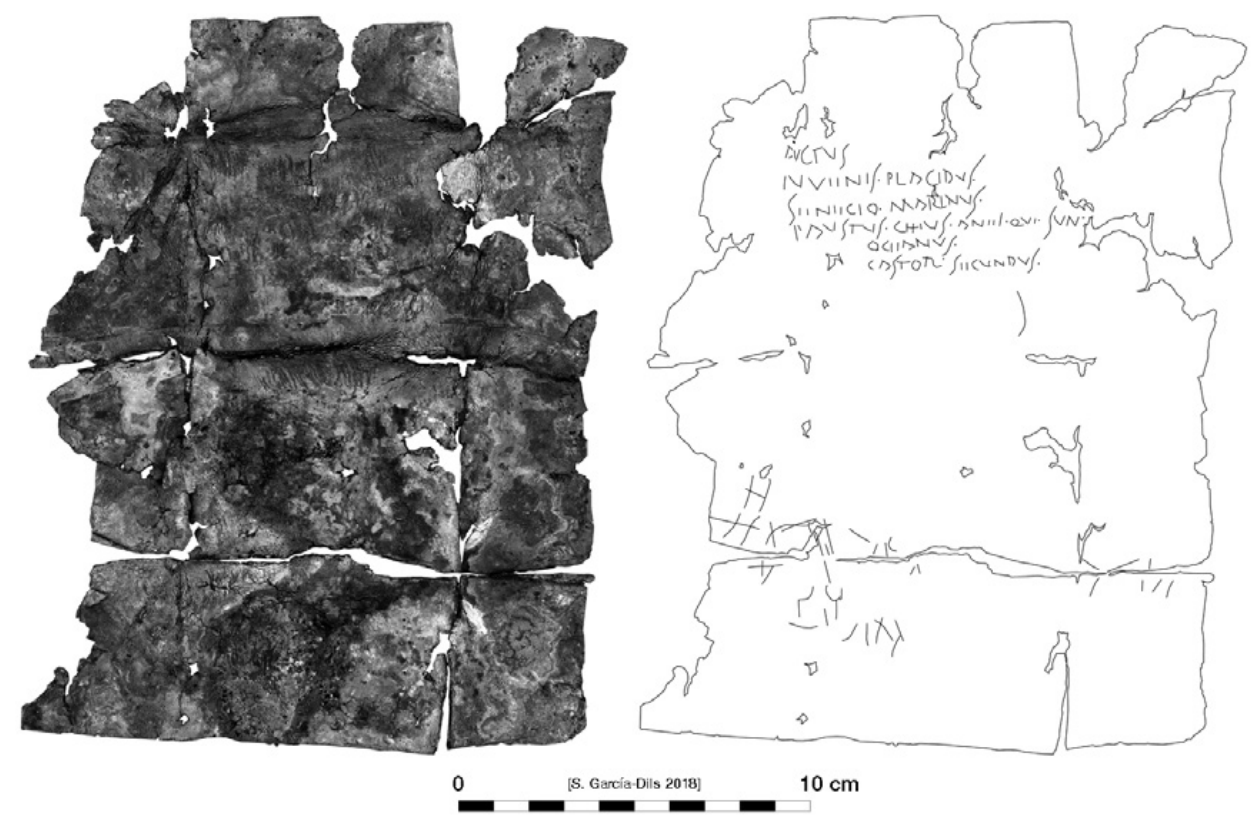

Fig. 7: Defixio C (S. García-Dils).

una misma mano ${ }^{29}$. También guarda estrecha relación tipológica con nuestra pieza la defixio judicial de Celti, que contiene fórmulas todavía más expresivas y rebuscadas: "Marcel(I) us Valerius mutus tacitus siet / adversus C. Licinio Gallo. Qu<em>admodum / rana sene (!) lingua muta tacita est, sic Marcellus mutus tacitus debilitatus siet / adv<e >rsus L[i] cinio Gallo" (AE 2012, $740=$ HEp 2012, 438; Stylow, 2014; Kruschwitz, 2016).

En la tabella que se presenta ahora se nombra explícitamente a los testigos como tales (testes), sin que haya que deducirlo del contexto, como es usual en este tipo de documentos epigráficos, lo que se registra aquí por primera vez. Sin embargo, no se limita a requerir la mudez para ellos, como se ha visto en los ejemplos precedentes, sino que además se pide que una serie de personajes, de los que se ha perdido el nombre, yazgan exánimes -"[ia]ceat exanimis"-. Al respecto, hay que señalar la presencia aquí de mujeres, ya que la expresión "yazga muda" -"muta iaceat" - indica claramente que se alude a un personaje femenino, protagonista en el proceso judicial, lo mismo que en las otras dos siguientes menciones de "muta".

\section{LA DEFIXIO C}

De nuevo, se trata de una tabella de plomo de forma irregular, aunque originalmente debió de ser rectangular. Aquí, en el ángulo inferior derecho es donde se puede apreciar mejor el recorte original. Tiene unas dimensiones de $17,24 \mathrm{~cm}$ de ancho y 20,98 cm de alto, con $2 \mathrm{~mm}$ de grosor máximo (Fig. 7). En este

${ }^{29}$ Cf. los rasgos paleográficos, sintetizados en Fig. 9, con Ventura, 1996: Fig. 1 y Fig. 2. 
caso, eran dos los clavos que atravesaban la lámina que, al estar doblada, presenta hasta seis orificios. El texto inscrito es el que sigue:

AVCTVS

IVVIINIS · PLACIDVS ·

SIINIICIO - MARINVS .

FAVSTVS $\cdot$ CHIVS $\cdot$ ANIII $\cdot$ QVI $\cdot$ SVN+ OCIIANVS .

CASTOR · SIICVNDVS ·

Auctus, / Iuvenis, Placidus, / Senecio, Marinus, / Faustus, Chius, An<na>ei qui sunt / Oceanus, / Castor, Secundus.

En esta ocasión, el texto está escrito de izquierda a derecha, con una cuidada caligrafía, interlineado homogéneo y correcta ordenación de los caracteres, que apenas se deformaron para adaptarse a las irregularidades del soporte. La deficiente conservación de la tabella dificulta no poco la lectura, dadas las deformaciones y pérdidas sufridas que experimentó al ser doblada y doblemente perforada, aunque afortunadamente estas circunstancias no han supuesto la pérdida de prácticamente nada del texto. En I. 1, los tres primeros caracteres de Auctus están deformados y desplazados de su ubicación original por los dobleces realizados a la tabeIla. Lo mismo en I. 2, en Iuvenis, en I. 3, en Senecio, y en I. 4, en Faustus. En esta I. 4, se grabó una i longa con la clara intención de diferenciarla de la e de doble trazo precedente (Fig. 8); más adelante, en + , se reconoce parte de una línea vertical y otra horizontal sobre ella, compatibles con una $t$, que habría sido afectada por la inserción de un clavo. Las II. 5 y 6 presentan mayor margen a la izquierda, para salvar una zona más irregular

30 También puede ser usado en femenino, como es el caso de la esclava Iuvenis, de Mediolanum (CIL V 5959). de la superficie de la tabella. Los caracteres presentan una altura uniforme de entre 3,0 y $3,5 \mathrm{~mm}$, con $s$ que llegan a los $10 \mathrm{~mm}$. El interlineado varía, adaptándose a las irregularidades del soporte, entre 3,0 y 5,0 mm. Presenta interpunciones circulares detrás de todas las palabras, salvo en I. 1 y, no sabemos, en I. 4, tras sunt, pues el orificio del mencionado clavo sigue a la $t$. Teniendo en cuenta la corrección y pericia del defigens, no está claro por qué el primer nombre, Auctus, aparece en I. 1 en solitario, lo mismo que Ocenanus en I. 5. El resto de los cognomina en las II. 2, 3, 4 y 6 se distribuyen ordenadamente en dos columnas.

El texto contiene un listado de cognomina masculinos de individuos de extracción servil, todos ellos en caso nominativo. Solamente en I. 4 se aparta de este patrón, con un nomen en plural, $A n<n a>e i$, seguido de la expresión qui sunt.

Auctus (I. 1). Se repite aquí el último de los cognomina de la defixio B (vid. supra).

Iuvenis (I. 2). Nombre utilizado en general en masculino ${ }^{30}$ (Kajanto, 1965: 78, 300; Abascal, 1994: 393-394; Lörincz, 1999: 211). Disponemos de un par de paralelos en Baetica, Q. Ennius Iuvenis, en una inscripción funeraria de procedencia desconocida, conservada en el Museo Provincial de Jaén (CIL II2/5, $53=$ CILA III.2, $630=$ HEp 1995, 534); otro luvenis en Almadén (HEp 2012, 269). En Hispania Citerior, contamos con L. Terentius Iuvenis, de Xèrica $\left(C / L\left\|^{2} / 14,269=C I L\right\|\right.$ 4012); [-] Geminius Q. f. Gal(eria) Iu(v) enis, de Saguntum (CIL II²/14, 619); en la misma ciudad, también un luvenis formando parte de un listado de nombres de personajes de condición servil ( $C / L \|^{2} / 14$, 


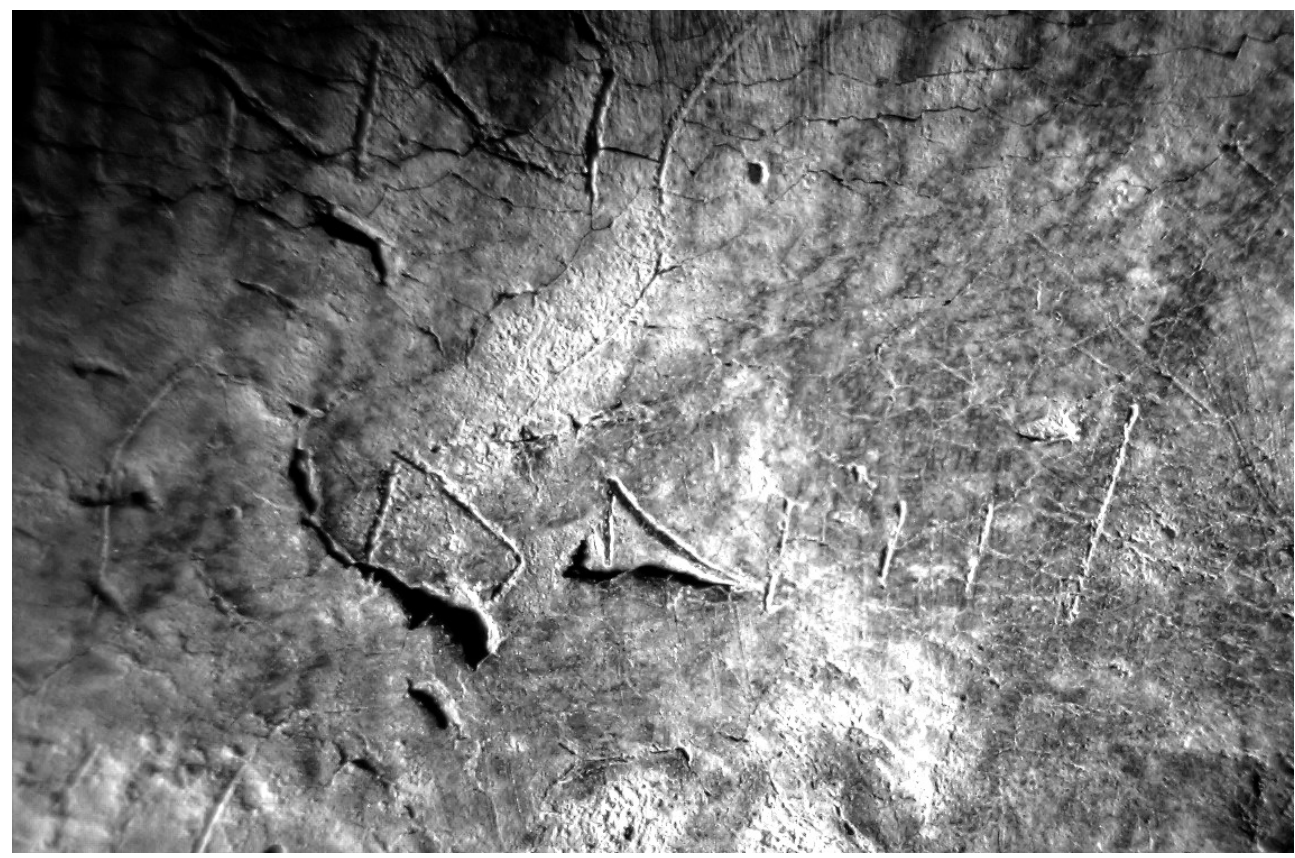

Fig. 8: Detalle de la I. 4 de la defixio C, tomado con microscopio digital (S. García-Dils).

$525=$ CIL II 3938), y el posible liberto [Pop]illius [--- I] HEp 2002, 132 = AE 1986, 447).

Placidus (I. 2). Cognomen de varón especialmente común en Hispania (Kajanto, 1965: 18, 262; Abascal, 1994: 460461; Lörincz, 2000: 144). En Baetica, Iunius Placidus, de Ugultunia (CIL II 1033); Q. Vinnius Q. I. Placidus, de Iulipa (CIL II2/7, 912); Q. Publicius Placidus, de Obulco (HEp 2009, 170 = AE 2013, 838). Una Fannia Placida en Corduba (CIL II2/7, 457).

Senecio (I. 3). Nombre masculino (Kajanto, 1965: 301; Abascal, 1994: 503; Lörincz, 2002: 65). Nos han Ilegado varios testimonios béticos, entre los que cabe mencionar el IVvir Fab(ius) Senecio, de Saepo (CIL II 1340 = IRPCádiz 540); M. Fulvius
Senecio, de Aratispi (CIL II/2/5, $733=C / L$ II 2056); L. Calpurnius Senecio, de Anticaria (CIL II/2/5, $754=$ CIL II $2050=$ AE 2012, 732); L. Virrius L. f. Pap(iria) Senecio, del cortijo Benavides, cerca de Astigi $(C / L ~ I I / 5,1286=$ CILA II.4, 1244 = HEp 1994, 681b = HEp 1996, 870). También se ha preservado una mención en Baetica del cónsul Q. Cornelius Gal(eria) Senecio Annianus, en una inscripción de Carteia (CIL II 1929 = IRPCádiz 85).

Marinus (l. 3). Cognomen de varón bien representado en Hispania (Kajanto, 1965: 81, 308; Abascal, 1994: 415; Lörincz, 2000: 58). Contamos con algunos ejemplos en Baetica, como un Marinus de Ventas de Zafarraya $(\mathrm{CIL} \mathrm{II} / 5,716=$ CILA IV 121 = HEp 1990, 413); otro personaje de este nombre, nombrado como amo de un escla- 
vo, en Minas de Riotinto (CILA I, $34=A E$ 1965, 299); otro Marinus en Siarum (CILA II.3, $944=H E p$ 1990, $644=A E$ 1988, 715). También se puede mencionar al emeritense L. Lucretius Marinus, que aparece como dedicante en una inscripción de Ugultunia (CIL II 1002 = HEp 1997, 25);

Faustus (I. 4). Aparece de nuevo este frecuente cognomen, que ya se ha visto en la defixio A (vid. supra).

Chius (I. 4). Nombre masculino de origen griego (Xîos - Fraser y Matthews, 19872008: s.v.; Abascal, 1994: 326; Solin, 1996: 369-370; Lozano, 1998: 56; Lörincz, 1999: 53). La mitad de los conocidos en Roma son esclavos o libertos (Solin, 2003: 647-648). En Hispania contamos con sendos paralelos, ambos localizados en Baetica, A. Firmius Apates lib. Chius, de Hispalis (CIL II 1227 = CILA II.1, 67), y L. Annius Chius, de Caelia (IRPCádiz 544 = HEp 1996, 252 = $A E$ 1975, 499). Cabe mencionar, en su versión femenina, a Iulia C(h)ia, de Gades (CIL II 1826 = IRPCádiz 222).

An<na>eus (I. 4, Fig. 8). En la forma ANEI podemos reconocer, en nominativo plural, el nomen An<na>eus (Abascal, 1994: 76; Lörincz y Redö, 1994: 116). En Corduba, nos han llegado testimonios epigráficos de Annaeus Vernaculus (CIL II2/7, $280=$ HEp 1989, 257), así como de L. Annaeus L. [- ---] y Annaea L. I. Prima (CIL II2/7, 406); también contamos con una inscripción del patriciense $C n$. Annaeus Natalis, de Tucci (CIL II2/5, $66=$ CILA III.2, 452 = HEp 1995, 478 = AE 1965, 80). Fuera de la capital, en Baetica está documentada Ann[e]ia M. f. Aciliana, en Celti (HEp 2005, 321 = $A E$ 2004, 751). Por su par- te, en Hispania Citerior, tenemos a C. Anneus (CIL II 4970, 70) y a Annaeus [Se] cundus (CIL II $4118=$ CIL II2/14, 979), ambos de Tarraco, y a C. Anneius, de Ibiza (HEp 1990, $58=A E$ 1990, 641). Con una grafía similar, sabemos de Aneius Caecilius, de Danilo Gornje (ILJug I, 175); M. Aneius M. I. Rufio, de Luceria -aunque el resto de los personajes nombrados aparece inscrito con doble $n-(A E 1983$, 220); Aneus, de Roma (CIL XV 6021).

Oceanus (I. 5). Nombre masculino con connotaciones mitológicas o geográficas (Kajanto, 1965: 216, 339; Lörincz, 2000: 109). Para este antropónimo de origen griego, que se popularizó como cognomen latino, contamos con un único testimonio hispano, Oceanus M. I., de Villar de Cañas (Abascal, 2013: 24 y Fig. 10; AE 2013, 903).

Castor (I. 6). Cognomen de varón de inspiración mitológica (Káotwp - Fraser y Matthews, 1987-2008: s.v.; Abascal, 1994: 319; Solin, 1996: 337; Lozano, 1998: 52; LörinCZ, 1999: 42; Solin, 2003: 537-539). Nos ha llegado un paralelo bético, $M$. Aur(elius) Castor, de Lebrija (CIL II 6338d $=E E$ VIII.2, $278=C I L A ~ I I .3,996=H E p$ 1997, 861). En Lusitania, un Castor aparece como dueño de un alfar en Abelterium $(H E p$ 2009, $580=A E$ 2010, $646=A E$ 2014, 573); otro caso en un grafito tardío, de Ossonoba (IRCP 17 = HEp 2006, 496). En Hispania Citerior, en una marca sobre tegula (IRVT II 133 = IRVT I 115b); Atilius Castor, de Huerta del Rey, en el territorium de Clunia (HEp 1990, 113).

Secundus (I. 6). Cognomen masculino, uno de los más frecuentes en Hispania (Kajanto, 1965: 73-78, 292; Duthoy, 1989; 
Abascal, 1994: 500-501; Lörincz, 2002: 59-61). Para Corduba solamente conocemos un paralelo masculino, la inscripción funeraria del patriciense $P$. Aemilius Secundus, localizada en Astigi (CIL II/2/5, $1314=$ HEp 1998, 407). Varios paralelos cordubenses para la forma femenina, entre los que se puede mencionar la inscripción funeraria de Persia L. f. Secunda (CIL II2/7, 392 = CIL II 2247); también Maia P. f. Secunda $\left(C / L I^{2} / 7,485=A E 1978\right.$, 419).

De nuevo se trata de una lista de nombres, por lo que su interpretación como defi- $x i o$, como en el caso de la primera presentada en este trabajo, se deduce de su contexto y el soporte empleado. Se puede reconocer además una serie de trazos en el cuadrante inferior izquierdo de la tabella, que no parecen corresponder a caracteres, por lo que quizá cabría interpretarlos como signa magica.

En cuanto a la expresión An<na>ei qui sunt, dado que se encuentra centrada respecto al resto del texto y desplazada a la derecha, se puede entender que se refiere al conjunto de los cognomina presentes; es decir, o bien se trataría de toda una serie de conliberti que compartirían el nomen An<na>eus, recibido

\begin{tabular}{|c|c|c|c|c|c|c|}
\hline $\mathrm{D}$ & $\mathrm{N}^{0}$ & COGNOMEN & ORIGEN & FORMA DOCUMENTADA & \multicolumn{2}{|c|}{$0^{\prime \prime} / 9$} \\
\hline A & 1 & Auge & AüYn & Auge & & 9 \\
\hline & 2 & Glucera (= Glycera) & 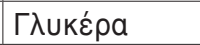 & Glucerae & & $q$ \\
\hline & 3 & Sabbis & semítico & Sabbinis & & 9 \\
\hline & 4 & Faustus & latino & Fausti & $\sigma^{\prime \prime}$ & \\
\hline & 5 & Fortunata & latino & Fortunatae & & $q$ \\
\hline & 6 & Nicocles & Nıкок入nิৎ & Nicoclis & $\sigma^{\prime \prime}$ & \\
\hline & 7 & Prima & latino & Primae & & 9 \\
\hline & 8 & Niceros & 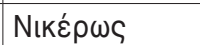 & Nicerotis & $\sigma^{\prime \prime}$ & \\
\hline & 9 & Macedo & Макєঠб́́v & Macedonis & $\sigma^{\prime \prime}$ & \\
\hline & 10 & Ingenuus & latino & Ingenui & $\sigma^{\prime \prime}$ & \\
\hline & 11 & Galla & latino & Gallae & & 9 \\
\hline & 12 & Philargurus (= Philargyrus) & Фı入ápүupos & Philarguri & $\sigma^{\prime \prime}$ & \\
\hline & 13 & Dionusius (= Dionysius) & Aıvúøios & Dionusi & $\sigma^{\prime \prime}$ & \\
\hline & 14 & Chiteris (= Cytheris) & Künpís & Chiteridis & & 9 \\
\hline & 15 & Nico o Nicon & 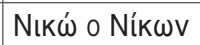 & Niconis & $\sigma^{\circ}$ & \\
\hline & 16 & Auge & AüYn & Augenis & & 9 \\
\hline & 17 & Chrestus & Хрйбтос & Chresti & $\sigma^{\infty}$ & \\
\hline & 18 & Maritumus & latino & Maritumi & $\sigma^{\circ}$ & \\
\hline & 19 & Basilius & 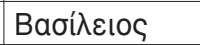 & Basili & $\sigma^{\circ}$ & \\
\hline & 20 & Mageia & céltico & Mageiae & & $q$ \\
\hline$B$ & 21 & $P(h)$ ronimus & Фрovímos & Promimus & $\sigma^{\circ}$ & \\
\hline & 22 & Stephanus & 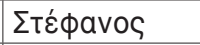 & Stephanus & $\sigma^{\circ}$ & \\
\hline & 23 & Primus & latino & Primus & $\sigma^{\circ}$ & \\
\hline & 24 & Auctus & latino & Auctus & $\sigma^{\pi}$ & \\
\hline C & 25 & Auctus & latino & Auctus & $\sigma^{\prime \prime}$ & \\
\hline
\end{tabular}




\begin{tabular}{|l|l|l|l|l|l|l|}
\hline D & $\mathbf{N}^{\circ}$ & COGNOMEN & ORIGEN & FORMA DOCUMENTADA & \multicolumn{2}{|c|}{$\sigma^{\prime \prime} /$ \$ } \\
\hline & 26 & luvenis & latino & luvenis & $\sigma^{\prime \prime}$ & \\
\hline & 27 & Placidus & latino & Placidus & $\sigma^{\prime \prime}$ & \\
\hline & 28 & Senecio & latino & Senecio & $\sigma^{\prime \prime}$ & \\
\hline & 29 & Marinus & latino & Marinus & $\sigma^{\prime \prime}$ & \\
\hline & 30 & Faustus & latino & Faustus & $\sigma^{\prime \prime}$ & \\
\hline & 31 & Chius & Xîos & Chius & $\sigma^{\prime \prime}$ & \\
\hline & 32 & Oceanus & latino & Oceanus & $\sigma^{\prime \prime}$ & \\
\hline & 33 & Castor & Káotwp & Castor & $\sigma^{\prime \prime}$ & \\
\hline & 34 & Secundus & latino & Secundus & $\sigma^{\prime \prime}$ & \\
\hline
\end{tabular}

Tabla resumen de los cognomina contenidos en las defixiones

de su patrono, o bien serían esclavos propiedad de un $A n<n a>e u s^{31}$. La presencia de este nomen nos lleva necesariamente a traer a colación la familia cordubense de los Annaei, con miembros tan ilustres como L. Annaeus Seneca padre (PIR A 616), L. Annaeus Seneca hijo (PIR ${ }^{2}$ A 617) y $M$. Annaeus Lucanus (PIR A 611). La cercanía cronológica de estos personajes a las fechas en las que se inscribió la defixio permitiría sugerir algún tipo de relación con alguno de ellos 0, por lo menos, con su familia, obviamente solamente a título de sugerencia.

\section{NOTAS FINALES}

El hallazgo de este nuevo sector funerario viene a completar la visión que hasta este momento se tenía de la Necrópolis Septen-

31 Una expresión similar, pero en contexto funerario, se constata por ejemplo en una inscripción de Terracina, "Monumentum et ossuar(ium) / et ustrinum / CC(aiorum) Iuliorum Faustorum et / eorum qui sunt" (CIL X 6368).

32 Destacando en este sentido la construcción del edificio que ocupaba el solar antes del comienzo de las intervenciones en el mismo y, sobre todo, la acción de la construcción de los muros pantalla y algunas fosas llevados a cabo durante el primer Seguimiento Arqueológico. trional de Colonia Patricia, un espacio que se vislumbraba perfectamente planificado y lotizado desde un primer momento, tal y como se intuía en las intervenciones llevadas a cabo en la antigua fábrica de la Constancia (Vaquerizo et alii, 2005) o en la Avenida de las Ollerías (López, 2006), donde se documentaron varias vías funerarias a las que abrían fachada diferentes recintos funerarios. A diferencia de lo que ocurrió en esos sectores, con una ocupación mucho más prolongada en el tiempo -llegando, al menos, al siglo II d.C.- y con una reutilización de los espacios, la necrópolis de Llanos del Pretorio se vió afectada por una riada o arroyada que la cubrió por completo. Este acontecimiento, con el añadido de que la zona no volvió a ser usada como lugar de enterramiento en época romana, ha provocado que hasta nuestros días haya llegado una instantánea de cómo se articulaba esta Necrópolis Septentrional en los comedios del siglo I d.C., estampa apenas alterada por la acción de algunas interfacies fechadas tanto en época islámica califal -siglo $X$ d.C.- como, sobre todo, en época contemporánea ${ }^{32}$.

El excepcional estado de conservación de la necrópolis, consecuencia de las circunstancias arriba señaladas, nos ha permitido acer- 
carnos al mundo funerario y todo lo que ello conlleva de una forma que hasta el momento no se había llevado a cabo en la capital de la Bética, a lo que ha contribuido de un modo decisivo la puesta en marcha de un proyecto multidisciplinar ${ }^{33}$ del que este este trabajo viene a ser uno de sus primeros frutos. La localización de estas tres defixiones no viene sino a refrendar estas afirmaciones, pues se trata de las primeras tabellae documentadas en Córdoba que cuentan con un contexto arqueológico preciso, pues las que se conocían hasta este momento se habían encontrado de forma casual (CIL II2/7, 250, CIL II2/7, 251, CIL II/7, 251a y C/L II/2/7, 252).

El contexto arqueológico inmediato de las tres defixiones presentadas en las líneas precedentes no nos permite establecer una relación directa con posibles aôroi -muertos prematuros- o biaiothánatoi -fallecidos de forma violenta, incluyendo suicidas, ajusticiados, asesinados, caídos en combate y muertos por accidente o enfermedad- (Ogden, 2002: 146-178; Alfayé, 2009: 184187; Sánchez Natalías, 2012: 117-119), ya que contamos con la dificultad que supone el que se trate de enterramientos de incineración, donde son mucho más difíciles de identificar las circunstancias de la muerte de los finados. Solamente en el caso de la defixio A, relacionada con el Enterramiento 7 , en el que se localizó un individuo infantil (0-6 años), cremado, podríamos establecer la posibilidad de la vinculación con una muerte prematura $^{34}$.

Formalmente, las tres se encuentran inscritas sobre láminas de plomo, tal como se prescribe repetidamente en Ios PGM (Sánchez Natalías, 2011). Solamente la B es explícitamente una defixio, mientras que para la A y la C, como ya se ha expuesto, podemos deducir este carácter precisamente a partir del soporte ${ }^{35}$, además de su contexto, así como la técnica de escritura en la primera de ellas. Por otra parte, solamente la B y la C presentan perforaciones de clavo.

Sin duda, una de las aportaciones de las defixiones presentadas radica en el amplio listado de cognomina de individuos de extracción servil que aportan ${ }^{36}$. Algunos aparecen documentados por primera vez en Baetica, como Glucera (= Glycera), Macedo, Chiteris (= Cytheris), Maritumus y Basilius defixio A-; Pronimus (= Phronimus) -defixio B-; Oceanus -defixio C-. También los hay atestiguados por primera vez en Hispania, ya sea en términos absolutos, como Sabbis y Mageia -defixio A-, o con esta grafía concreta, como Glucera, Dionusius, Chiteris -defixio A- y Pronimus -defixio B-. Por fin, en un caso, nos encontramos con el primer testimonio en el conjunto del Imperio, Nicocles, hasta ahora solamente registrado en su versión femenina, Nicoclea-defixio A-.

En cuanto a la cronología de las tres defixiones, sus características formales, algún posible arcaísmo lingüístico ${ }^{37}$ y el estudio de

33 Vid. supra n. 1.

34 Los Dres. Manuel Polo Cerdá y Elisa García Prósper han sido los responsables del estudio de los restos óseos humanos dentro del proyecto "Sepulcretum Llanos del Pretorio". Los autores agradecen su amabilidad por adelantarles los primeros resultados de su trabajo.

35 Los listados de cognomina sobre soporte plúmbeo que aparecen en las defixiones A y C, sin ningún tipo de maldición explícita, son análogos en su tipología a los que encontramos, también en Corduba, en CIL II2/7, 251 y CIL II2/7, 252.

36 Sobre el estudio de los cognomina en relación con la caracterización de la extracción social de los individuos que los portan, vid. Duthoy, 1989.

37 Por ejemplo, la transcripción de la aspirada griega por su correspondiente oclusiva sorda en latín en el caso 


\begin{tabular}{|c|c|c|c|}
\hline & defixio A & defixio B & defixio C \\
\hline A & $\begin{array}{l}A \wedge A \wedge \wedge \wedge \wedge \wedge A \wedge \wedge \\
\Lambda \wedge / A \wedge\end{array}$ & $\begin{array}{l}A \lambda \lambda \wedge \lambda \wedge \lambda \lambda \lambda \lambda A \\
\lambda \wedge \lambda\end{array}$ & $\lambda \gg \gg \gg \gg$ \\
\hline B & $B B \beta$ & & \\
\hline C & ()) () & $c<c$ & $l(c c c c$ \\
\hline D & $\| \ll$ & & $D D$ \\
\hline E & |||||||||||||||||||||||| $\mid$ & \|\|\|\|\|\|\|\|\|\| & 11111111111 \\
\hline $\mathrm{F}$ & 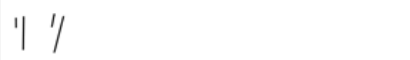 & & 1 \\
\hline G & 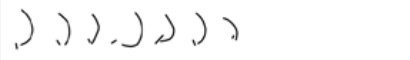 & & \\
\hline $\mathrm{H}$ & $\mathrm{HHH}$ & $H$ & $H$ \\
\hline 1 & 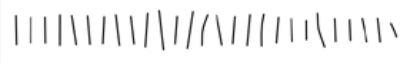 & 111111111 & $111111 / 1$ \\
\hline $\mathrm{L}$ & \lrcorner$\lrcorner\lrcorner\lrcorner\lrcorner\lrcorner$ & & L \\
\hline M & $M M M M M$ & $M_{M M M M} M M M M$ & $N$ \\
\hline $\mathrm{N}$ & МиИииИии иии & $\operatorname{MnNH}$ & $N N N N N N N$ \\
\hline $\mathrm{O}$ & 000000 & 90 & 000 \\
\hline $\mathrm{P}$ & $\rho P$ & $P P P$ & p \\
\hline Q & & & 2 \\
\hline $\mathrm{R}$ & イ イЛイイイイ イ & $R R$ & $R \pi$ \\
\hline S & 322323232223 & & $\iint$ \\
\hline $\mathrm{T}$ & T†十面 & 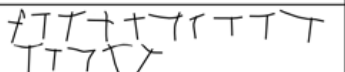 & $T T T$ \\
\hline V & $\vee \vee \vee \vee \vee \vee \vee \vee \vee$ & $\begin{array}{l}\vee V Y \vee V \vee V Y \vee V \vee \\
\text { VVマX }\end{array}$ & $\begin{array}{l}\text { VソVVVVV } \\
\cup \vee V \vee V \vee\end{array}$ \\
\hline$x$ & & $x X$ & \\
\hline
\end{tabular}

Fig. 9: Caracteres completos que aparecen en las defixiones, por orden de aparición (S. García-Dils).

de nombres orientales, como se puede ver en Pro'n'imus / $P<h>r 0^{\prime} n^{\prime} i m u s$ en la defixio $B$, o en el caso de Mageia en la defixio A, si se acepta que se quiso escribir aquí Magia, usándose el diptongo ei en lugar de i (Díaz 2008: 24-25). la paleografía (Fig. 9) sugieren, por comparación con otras tabellae béticas, como las cordobesas CIL II2/7, 250, CIL II/2/7, 251, CIL II/7, 251a y CIL II/2/7, 252, la de Celti 
$($ AE 2012, $740=$ HEp 2012, 438; STYLOW 2014) y la de Iliturgi (CILA II.4, $1249=$ HEp 1995, $695=$ HEp 1999, $503=$ HEp 2008, $83=A E 1993,1008=A E 1995,770=A E$ 1999, $894=A E 2010,108)^{38}$, una fecha en la segunda mitad del siglo I a.C ${ }^{39}$. Sin embargo, como se ha visto, el estudio de los ma- teriales arqueológicos aconseja situarlas más adelante cronológicamente, por lo menos en época de Tiberio, lo que nos lleva a interrogarnos sobre la datación de las defixiones precitadas, carentes de contexto claro, así como sobre el consiguiente conservadurismo en la escritura cursiva que se deduciría de ello.

\section{BIBLIOGRAFÍA}

ABASCAL PALAZÓN, J. M. (1994): Los nombres personales en las inscripciones latinas de Hispania, Murcia.

- (2013): "Cuestiones epigráficas del conventus Carthaginiensis (Hispania Citerior), con algunas contribuciones póstumas de Géza Alföldy", en LÓPEZ VILAR, J. (ed.), Actes. 1er. Congrés Internacional d'Arqueologia i Món Antic. Govern i societat a la Hispània romana. Novetats epigràfiques, Tarragona, pp. 13-34.

ALFAYÉ VILLA, S. (2009): "Sit tibi terra gravis: Magical-ritual Practices against Restless Dead in the Ancient World", en MARCO, F., PINA, F. y REMESAL, J. (eds.), Formae mortis. El tránsito de la vida a la muerte en las sociedades antiguas, Barcelona, pp. 181-214.

AUDOLLENT, A. (1904): Defixionum tabellae quotquot innotuerunt tam in graecis Orientis quam in totius Occidentis Partibus praeter Atticas in Corpore Inscriptionum Atticorum editas, Paris.

BAENA ALCÁNTARA, M. D. (1989): “Intervención Arqueológica de Urgencia en Avenida de las Ollerías $n^{0} 14,1^{\text {a }}$ fase (Córdoba)", AAA 1989, vol. III, pp. 135-138.

BARTA, A. (2017): "The Siscia Curse Tablet from a Linguistic Point of View. A New Autopsy", Graeco-Latina Brunensia, 22, pp. 23-41.

BLÄNSDORF, J. y SCHOLZ, M. (2011): "Fluchtäfelchen: Verfluchter Prozessgegner. Zwei defixiones aus dem 'Älteren Praunheimer Gräberfeld'", en FASOLD, P. (ed.), Die Bestattungsplätze des römischen Militärlagers und Civitas-Hauptortes
Nida (Frankfurt am Main-Heddernheim und -Praunheim), Frankfurt a. M., pp. 66-77.

CLAPÉS SALMORAL, R., CASTILLO PÉREZ DE SILES F., TEJEDOR GARCÍA, U. y RUBIO VALVERDE, M. (2015): "El sarcófago romano de los Jardines de la Agricultura (Córdoba). Análisis arqueológico y antropológico", Romula, 15, pp. 255-282.

DÍAZ ARIÑO, B. (2008): Epigrafía latina republicana de Hispania (ELRH), Barcelona.

DUTHOY, R. (1989): “Cognomen est omen? Quelques jalons pour une anthroponymie sociale du monde romain", en MACTOUX, M. M. y GENY, E. (eds.), Mélanges Pierre Lévêque. Tome 2: Anthropologie et société, Besançon, pp. 183-205.

FRASER, P. M. y MATTHEWS, E. (eds.) (19872008): Lexicon of Greek Personal Names, Oxford.

GAGER, J. G. (1992): Curse Tablets and Binding Spells from the Ancient World, New York-Oxford.

GIORCELLI BERSANI, S. (2007): "Nuovi documenti epigrafici dalla Valle Sesia (VC) per la storia della romanizzazione della Cisalpina", Epigraphica, 69, pp. 117-147.

ISINGS, C. (1957): Roman Glass from dated finds, Gröningen-Djakarta.

38 Este documento epigráfico, proveniente en realidad de Mengíbar, ha venido adscribiéndose erróneamente desde su publicación inicial a Carmona (HEp 2008, 83).

39 Para una visión general sobre las defixiones republicanas hispanas, vid. Díaz 2008: 72-73. 
KAJANTO, I. (1965): The Latin Cognomina, Helsinki.

KRUSCHWITZ, P. (2016): "A Frog in the Throat: À Propos AE 2012.740 = ZPE 181 (2012) 150", ZPE 198, 162-163.

LÓPEZ CASTRO, J. L. y BELMONTE MARÍN, J. A. (2012): "Pervivencias de la antroponimia fenicia en época romana en la península Ibérica", en MORA SERRANO, B. y CRUZ ANDREOTII, G. (coord.), La etapa neopúnica en Hispania y el Mediterráneo centro occidental: identidades compartidas, Sevilla, pp. 141-164.

LÓPEZ JIMÉNEZ, A. (2006): Informe y Memoria. Parcela 4 del Plan Especial SC-2a, 06. Informe administrativo inédito depositado en la Delegación de Cultura de Córdoba.

LÖRINCZ, B. (1999): Onomasticon Provinciarum Europae Latinarum (OPEL). Vol. Il: Cabalicius Ixus, Wien.

- (2000): Onomasticon Provinciarum Europae Latinarum (OPEL). Vol. III: Labareus - Pythea, Wien.

(2002): Onomasticon Provinciarum Europae Latinarum (OPEL). Vol. IV: Quadratia - Zures, Wien.

LÖRINCZ, B. y REDÖ, F. (1994): Onomasticon Provinciarum Europae Latinarum (OPEL). Vol. I: Aba - Bysanus, Wien.

LOZANO VELILLA, A. (1998): Die griechischen Personennamen auf der iberischen Halbinsel, Heidelberg.

MARCO SIMÓN, F. (2010a): "Execrating the Roman Power", en GORDON, R. L. y MARCO SIMÓN, F. (eds.), Magical Practice in the Latin West. Papers from the International Conference held at the University of Zaragoza, Leiden-Boston, pp. 399-423.

- (2010b): "Muta Tacita en dos textos mágicos", SMSR, 76.1, pp. 101-115.

MELCHOR GIL, E. (1995): Vías romanas de la provincia de Córdoba, Córdoba.
MORENO ROMERO, L. (2007): "Santa Rosa. Un sector de la Necrópolis Septentrional de Colonia Patricia", Arqueología Cordobesa, 15, Córdoba.

OGDEN, D. (2002): Magic, Witchcraft, and Ghosts in the Greek and Roman Worlds. A Sourcebook, Oxford.

ORDÓÑEZ AGULLA, S. y GARCÍA-DILS DE LA VEGA, S. (e.p. 2018): "Tablillas de maldición en Andalucía. Magia, maleficios y conjuros en la religiosidad popular romana", Andalucía en la Historia.

PENCO VALENZUELA, R. (2007): Informe y Memoria de la Actividad Arqueológica Preventiva en Avenida Llanos del Pretorio № 1 y 3 (Córdoba). Informe administrativo inédito depositado en la Delegación de Cultura de Córdoba.

- (2008): Informe del Seguimiento Arqueológico en Avenida Llanos del Pretorio № 1 y 3 (Córdoba). Informe administrativo inédito depositado en la Delegación de Cultura de Córdoba.

PENCO VALENZUELA, F., MARFIL RUIZ, P. F., BLANES DELGADO, C. y BAENA ALCÁNTARA, M. D. (1993): "Resultados del estudio de la $\mathrm{Ne}$ crópolis romana excavada durante las dos fases de Intervención Arqueológica de Urgencia desarrolladas en la Avenida de las Ollerías № 14 de Córdoba", Antiquitas, 4, pp. 45-46.

PREISENDANZ, K. (1972): "Fluchtafel (Defixion)", $R A C, 8$, pp. 1-29.

RUIZ OSUNA, A. B. (2007): "La historiografía local como herramienta de reconstrucción del mundo funerario en Colonia Patricia Corduba", Spal, 16 , pp. 25-40.

SALINAS PLEGUEZUELO, E. (2015): "Nuevos hallazgos exhumados en una necrópolis romana del sector septentrional de Córdoba (España)", Nailos, 2, pp. 253-273.

SÁNCHEZ DE PRADO, M. D. (2018): La vajilla de vidrio en el ámbito suroriental de la Hispania romana. Comercio y producción entre los siglos IVII d.C., Alicante. 
SÁNCHEZ NATALÍAS, C. (2011): "Escribiendo una defixio: Los textos de maldición a través de sus soportes", ACD, XLVII, pp. 79-93.

(2012): "Muertos mágicos: Defixiones en contexto necropolitano", Antesteria, 1, pp. 117126.

(2014): “....ut illam ducas...'. Una nueva interpretación de la defixio contra Salpina", ZPE 191, pp. 278-281.

SOLIN, H. (1996): Die stadtrömischen Sklavennamen: ein Namenbuch. Teil 2. Griechische Namen, Stuttgart.

- (2003): Die griechischen Personennamen in Rom. Ein Namenbuch, Berlin-New York.

- (2009): "Nomi greci nel mondo romano", QuIRIOn, 2, pp. 61-84.

(2014a): "Iscrizioni giudaiche antiche a Fondi", en LACERENZA, G. (ed.), Gli ebrei a Fondi e nel suo territorio. Atti del convegno. Fondi, 10 maggio 2012, Napoli, pp. 33-48.

(2014b): "On the Presence of Jews in Ancient Latium", SCI, 33, pp. 243-254.

STYLOW, A. U. (2014): “¡Mudo como rana sin lengua! Nueva tabella defixionis de Celti (Peñaflor, Sevilla)", en FARMM. Fondo arqueológico Ricardo Marsal Monzón, Sevilla, pp. 211-215.
VAQUERIZO GIL, D. (2001): “Necrópolis Septentrional", en VAQUERIZO GIL, D. (ed). Funus Cordubensium. Costumbres funerarias en la Córdoba romana, Córdoba, pp. 124-125.

VAQUERIZO GIL, D., GARRIGUET MATA, J. A. y VARGAS CANTOS, S. (2005): La Constancia. Una contribución al conocimiento de la topografía y los usos funerarios en la Colonia Patricia de los siglos iniciales del Imperio, Arqueología Cordobesa, 11, Córdoba.

VAQUERIZO GIL, D. y SÁNCHEZ MADRID, S. (2008): "Entre lo público y lo privado. Indicatio Pedaturae en la epigrafía funeraria hispana", AEspA, 81, pp. 101-131.

VENTURA VILLANUEVA, A. (1996): "Magia en la Córdoba romana", AAC, 7, pp. 141-162.

VERSNEL, H. S. (1991): "Beyond Cursing: The Appeal to Justice in Judicial Prayers", en FARAONE, C. A. y OBBINK, D. (eds.), Magika Hiera. Ancient Greek Magic and Religion, New York-Oxford, pp. 60-106.

(2010): "Prayers for Justice, East and West: New Finds and Publications since 1990", en GORDON, R. L. y MARCO SIMÓN, F. (eds.), Magical Practice in the Latin West. Papers from the International Conference held at the University of Zaragoza, Leiden-Boston, pp. 275-354. 
